\title{
New insights into the heterogeneity of the Tagish Lake meteorite: Soluble organic compositions of variously altered specimens
}

\author{
Danielle N. SIMKUS (iD ${ }^{1,2,5^{*}}$, José C. APONTE (iD ${ }^{2,3}$, Jamie E. ELSILA (iD ${ }^{2}$, Robert W. HILTS ${ }^{4}$, \\ Hannah L. McLAIN ${ }^{2,3}$, and Christopher D. K. HERD ${ }^{1}$ \\ ${ }^{1}$ Department of Earth and Atmospheric Sciences, University of Alberta, Edmonton, Alberta T6G 2R3, Canada \\ ${ }^{2}$ Solar System Exploration Division, Code 691, NASA Goddard Space Flight Center, Greenbelt, Maryland 20771, USA \\ ${ }^{3}$ Department of Chemistry, Catholic University of America, Washington, DC 20064, USA \\ ${ }^{4}$ Department of Physical Sciences, MacEwan University, Edmonton, Alberta T5J 4S2, Canada \\ ${ }^{5}$ Present address: NASA Postdoctoral Program at NASA Goddard Space Flight Center, Greenbelt, Maryland 20771, USA \\ *Corresponding author. E-mail: danielle.n.simkus@nasa.gov
}

(Received 30 November 2018; revision accepted 09 February 2019)

\begin{abstract}
The Tagish Lake carbonaceous chondrite exhibits a unique compositional heterogeneity that may be attributed to varying degrees of aqueous alteration within the parent body asteroid. Previous analyses of soluble organic compounds from four Tagish Lake meteorite specimens (TL5b, TL11h, TL11i, TL11v) identified distinct distributions and isotopic compositions that appeared to be linked to their degree of parent body processing (Herd et al. 2011; Glavin et al. 2012; Hilts et al. 2014). In the present study, we build upon these initial observations and evaluate the molecular distribution of amino acids, aldehydes and ketones, monocarboxylic acids, and aliphatic and aromatic hydrocarbons, including compound-specific $\delta^{13} \mathrm{C}$ compositions, for three additional Tagish Lake specimens: TL1, TL4, and TL10a. TL1 contains relatively high abundances of soluble organics and appears to be a moderately altered specimen, similar to the previously analyzed TL5b and TL11h lithologies. In contrast, specimens TL4 and TL10a both contain relatively low abundances of all of the soluble organic compound classes measured, similar to TL11i and TL11v. The organic-depleted composition of TL4 appears to have resulted from a relatively low degree of parent body aqueous alteration. In the case of TL10a, some unusual properties (e.g., the lack of detection of intrinsic monocarboxylic acids and aliphatic and aromatic hydrocarbons) suggest that it has experienced extensive alteration and/or a distinct organicdepleted alteration history. Collectively, these varying compositions provide valuable new insights into the relationships between asteroidal aqueous alteration and the synthesis and preservation of soluble organic compounds.
\end{abstract}

\section{INTRODUCTION}

The Tagish Lake meteorite, hereinafter referred to as "Tagish Lake" (TL), is an ungrouped type 2 carbonaceous chondrite with compositional similarities to both CI and CM chondrites (Zolensky et al. 2002). The meteorite fell onto the surface of a frozen lake (Tagish Lake) in British Columbia on January 18, 2000 (Brown et al. 2000). The first Tagish Lake specimens were collected from the ice surface without direct hand contact within only a few days of the fall and have since been stored below $0{ }^{\circ} \mathrm{C}$ (Brown et al. 2000; Herd et al. 2016). These specimens, most of which are currently housed in the Subzero Facility for Curation of Astromaterials at the University of Alberta, are considered the most well-preserved carbonaceous chondrite samples available for study (Hilts et al. 2014; Herd et al. 2016). The low-temperature storage of these meteorite specimens aims to lower reaction rates, inhibit potential microbial growth, and retain intrinsic volatile organic species within the samples (Herd et al. 2016). In addition to its unique collection and curation conditions, Tagish Lake has an unusual mineralogical, petrological, and geochemical heterogeneity that has 
been attributed to varying degrees of aqueous alteration across the parent body asteroid (Herd et al. 2011; Alexander et al. 2014; Blinova et al. 2014a, 2014b; Hilts et al. 2014). Such variation within one single meteorite has not been observed before and affords the potential for an entirely new window into the chemical processes occurring in asteroid parent bodies.

Studies of several Tagish Lake fragments indicate that it is the most carbon-rich chondrite known to date (e.g., $5.8 \mathrm{wt} \%$ C; Grady et al. 2002), it has an exceptionally low bulk density $\left(1.6 \mathrm{~g} \mathrm{~cm}^{-3}\right.$; Zolensky et al. 2002) and a high porosity (30-40\%; Bland et al. 2004; Ralchenko et al. 2014), and is highly friable compared to other carbonaceous chondrite samples (Zolensky et al. 2002; Herd et al. 2016). The bulk mineralogy of the meteorite consists of olivine- and pyroxene-bearing chondrules, and isolated olivine and pyroxene grains and lithic fragments, set within a finegrained matrix of phyllosilicates (saponite and serpentine clays), sulfides, magnetite, and carbonates (Brown et al. 2000; Blinova et al. 2014a). The first investigation of soluble organic compounds for a pristine Tagish Lake specimen identified a suite of organic compounds, including aliphatic and aromatic hydrocarbons, amino acids, mono- and dicarboxylic acids, pyridine carboxylic acids, and sulfonic acids (Pizzarello et al. 2001). Subsequent organic analyses of Tagish Lake have focused primarily on four additional specimens-TL5b, TL11h, TL11i, and TL11v-which have been analyzed in detail for their mineralogical and chemical compositions (Herd et al. 2011; Glavin et al. 2012; Alexander et al. 2014; Blinova et al. 2014a, 2014b; Hilts et al. 2014). Mineralogical indicators for aqueous alteration (e.g., increasing replacement of chondrule glass by phyllosilicates in combination with relatively low porosity and a lower proportion of amorphous silicate material) suggest that the degree of aqueous alteration for these specimens is in the increasing order of TL5b $<$ TL11h $\ll$ TL11i (Herd et al. 2011). TL11v is a disaggregated sample and appears to be a physical mixture of several lithologies (Blinova et al. 2014a). On the basis of thermogravimetric analysis and infrared transmission spectroscopy, Gilmour et al. (2019) suggest that two additional specimens, TL4 and TL10a, are similar in degree of aqueous alteration to TL5b and TL11h. These authors find that the alteration within Tagish Lake is similar to the lesser altered members of the $\mathrm{CM}$ chondrites, and that Tagish Lake could be considered a type 1.6-2.0 ungrouped carbonaceous chondrite.

The soluble organic content of Tagish Lake and other carbonaceous chondrites provide a chemical record of the prebiotic synthesis reactions that took place throughout the early solar system during its formation and during subsequent parent body alteration. The origin and distribution of meteoritic organic compounds (e.g., amino acids, carboxylic acids, aldehydes, and ketones) are of interest for understanding the origin of life on Earth and the potential for life elsewhere, as these compounds are known precursors to several key biological molecules (Botta and Bada 2002; Sephton 2002; Glavin et al. 2018). Amino acids, the building blocks of protein, may be synthesized via the Strecker-cyanohydrin reaction (Peltzer et al. 1984). Through this reaction, an aldehyde or ketone reacts with ammonia in the presence of hydrogen cyanide $(\mathrm{HCN})$ to form an $\alpha$-amino acid. Other possible origins for meteoritic amino acids include the Michael-type addition of ammonia to unsaturated nitriles to yield $\beta$-amino acids (Cronin and Chang 1993), reductive amination of keto acids (Huber and Wächtershäuser 2003), and $\mathrm{CO}_{2}$ addition to amines (Hudson et al. 2009; Pietrucci et al. 2018). Aldehydes and ketones, the Strecker amino acid precursors, are also potentially structurally related to carboxylic acidsthe building blocks of lipids - through oxidation/ reduction reactions.

Amino acids, carboxylic acids, and other aliphatic and aromatic organic species have been previously detected in Tagish Lake samples, and their total abundances, distributions, and isotopic compositions have been shown to vary widely across the range of lithologies (Herd et al. 2011; Glavin et al. 2012; Hilts et al. 2014). In general, the samples more strongly affected by aqueous alteration (e.g., TL11i) have been shown to contain lower abundances of soluble organics, while the minimally/moderately altered samples (TL5b, TL11h) are relatively organic-rich. The amino acid compositions suggest that moderate aqueous alteration allows for optimal synthesis and/or preservation of amino acid molecules, and the compound-specific stable isotope ratios and enantiomeric compositions also appear to be influenced by the degree of aqueous alteration (Herd et al. 2011; Glavin et al. 2012; Hilts et al. 2014). These cause and effect relationships, however, are difficult to assess without a larger data set to evaluate.

In the present study, we have built upon the initial investigations of Tagish Lake by exploring the soluble organic content of three meteorite specimens previously unstudied in terms of organic matter (TL1, TL4, and TL10a), including compound-specific carbon isotope analysis of amino acids, solid-phase microextraction (SPME) analysis of carboxylic acids, and dichloromethane (DCM) solvent extractions for determining the aliphatic and aromatic content of the samples. This study also includes the first analysis of aldehydes and ketones in the Tagish Lake meteorite and 
their measured compound-specific carbon isotope compositions. The $\delta^{13} \mathrm{C}$ values reported here are discussed in comparison to the $\delta^{13} \mathrm{C}$ values of other compound classes in the meteorite to evaluate their origins and investigate potential formation relationships. By comparing our soluble organic data with previous analyses, we aim to place TL1, TL4, and TL10a into the previously described sequence of aqueous alteration, taking into consideration that the variability observed may not only reflect distinct alteration histories but also a heterogeneous distribution of key chemical precursor compounds across the parent body asteroid.

\section{MATERIALS AND METHODS}

\section{Chemicals and Reagents}

All glassware and tools were wrapped in aluminum foil and heated at $>450{ }^{\circ} \mathrm{C}$ for a minimum of $6 \mathrm{~h}$ before use in order to remove organic contamination. All vials were capped with PTFE-lined lids. Standards and reagents were purchased from Sigma-Aldrich and Fisher Scientific. Ultrapure water (Millipore Direct Q3 UV, 18.2 $\mathrm{M} \Omega, 3 \mathrm{ppb}$ total organic carbon; hereafter referred to as "water"), HPLC grade DCM, double-distilled $6 \mathrm{M}$ $\mathrm{HCl}$, and $O-(2,3,4,5,6$-pentafluorobenzyl) hydroxylamine hydrochloride (PFBHA) of $\geq 99.0 \%$ purity were used. Stock aldehyde/ketone, carboxylic acid, and amino acid solutions were prepared by mixing individual standards (97-99\% purity) in Millipore (18.2 M $\Omega$ ) or doubledistilled water. The $o$-phthaldialdehyde $/ N$-acetyl-Lcysteine (OPA/NAC) reagent used for amino acid derivatization was prepared by mixing $300 \mu \mathrm{L} 0.1 \mathrm{M}$ OPA in methanol, and then adding $670 \mu \mathrm{L} 0.1 \mathrm{M}$ sodium borate buffer $(\mathrm{pH} 9)$ and $30 \mu \mathrm{L} 1$ M NAC. A $0.1 \mathrm{M}$ hydrazine $\left(\mathrm{NH}_{2} \mathrm{NH}_{2}\right)$ solution was prepared by double vacuum distillation of anhydrous hydrazine ( $98 \%$ purity) and subsequent dilution in water. The ammonium formate buffer used for the liquid chromatography time-of-flight mass spectrometry (LCTOF-MS) analyses was prepared by $\mathrm{NH}_{4} \mathrm{OH}$ titration of a $50 \mathrm{mM}$ formic acid solution to $\mathrm{pH}$ 8. Details regarding the preparation of solutions and the sources of specific five-carbon (C5) amino acids used as standards are available in Glavin et al. (2006) and Glavin and Dworkin (2009).

\section{Tagish Lake Samples and Procedural Blanks}

Samples of three Tagish Lake specimens-TL1 (MET11611/P-1, original weight $157.8 \mathrm{~g} ; 7.1 \mathrm{~g}$ total mass removed), TL4 (MET11611/P-4, original weight $60.0 \mathrm{~g} ; 6.1 \mathrm{~g}$ total mass removed), and TL10a (MET11611/P-10a, original weight $111.0 \mathrm{~g} ; 5.6 \mathrm{~g}$ total mass removed)—were provided by the Meteorite Collection, Department of Earth and Atmospheric Sciences, University of Alberta. All meteorite subsampling was carried out in the Subzero Facility for Curation of Astromaterials at the University of Alberta. The facility consists of a purified Ar glove box located within a walk-in freezer $\left(<-10^{\circ} \mathrm{C}\right)$, providing an inert, low-temperature atmosphere for meteorite processing (Herd et al. 2016). All Tagish Lake subsamples were interior chips that did not contain any visual evidence of fusion crust. Individual Tagish Lake subsamples for amino acid analysis of TL1 (3.615 g) and TL4 (2.589 g) were shipped to NASA Goddard's Astrobiology Analytical Laboratory for powdering, extraction, and analysis (see the Aldehydes and Ketones: Identification, Quantification, and $\delta^{13} \mathrm{C}$ Analysis section). Subsamples for the amino acid analysis of TL10a (2.027 g), aldehyde and ketone analysis (TL1, $2.492 \mathrm{~g}$; TL4, 2.457 g; TL10a, $2.386 \mathrm{~g}$ ), carboxylic acid analysis (TL1, $0.490 \mathrm{~g}$; TL4, $0.500 \mathrm{~g} ;$ TL10a, $0.640 \mathrm{~g}$ ), and DCM extractions (TL1, $0.500 \mathrm{~g}$; TL4, $0.500 \mathrm{~g}$; TL10a, $0.582 \mathrm{~g}$ ) were powdered in the purified Ar glove box in the Subzero Facility for Curation of Astromaterials. For each type of soluble organic analysis, two procedural blanks were carried through the meteorite extraction procedure and sample work-up, unless noted otherwise. The procedural blanks included one solvent blank, containing no mineral component, and one mineral analog blank, consisting of $\sim 500 \mathrm{mg}$ of Allende meteorite powder or serpentine mineral powder (heated at $450{ }^{\circ} \mathrm{C}$ for $>6 \mathrm{~h}$ prior to the extraction step to drive off organics).

\section{Amino Acids: Identification, Quantification, and $\delta^{13} \mathrm{C}$ Analysis}

The TL10a specimen (see the Tagish Lake Samples and Procedural Blanks section) was extracted at the University of Alberta, as follows: The powdered sample was divided into four $\sim 0.5 \mathrm{~g}$ aliquots in glass vials. The glass vials were removed from the subzero purified $\mathrm{Ar}$ glovebox and kept cold until the powders were transferred to four $5 \mathrm{~mL}$ glass ampules. Of water, $2 \mathrm{~mL}$ was added to each ampule, the ampules were flamesealed, and the solutions were heated at $100{ }^{\circ} \mathrm{C}$ for $24 \mathrm{~h}$. The water extracts were centrifuged and the supernatants were combined. The residual solids were rinsed three additional times using $0.5 \mathrm{~mL}$ of water per rinse. The combined supernatants and rinses were evaporated to dryness using a rotary evaporator. The dried extract was then shipped to the NASA Goddard Astrobiology Analytical Laboratory for analysis, along with a dried procedural blank that was carried through the same procedure. The TL1 and TL4 meteorite chips 
(see the Tagish Lake Samples and Procedural Blanks section) were shipped directly to the NASA Goddard Astrobiology Analytical Laboratory for the water extraction and sample work-up, carried out as follows: TL1 and TL4 were powdered and homogenized using a mortar and pestle in a positive pressure ISO 5 HEPAfiltered laminar flow hood, and separated into $\sim 300 \mathrm{mg}$ portions. The total masses of the samples after powdering were: $3.525 \mathrm{~g}$ for TL1 and $2.537 \mathrm{~g}$ for TL4. Each portion was extracted at $100{ }^{\circ} \mathrm{C}$ for $24 \mathrm{~h}$ inside flame-sealed glass ampules each containing $1 \mathrm{~mL}$ of water. Extracts were separated from the residues by centrifugation, the residues were rinsed with water $(3 \times 0.5 \mathrm{~mL})$, and the rinses were combined with the aqueous portion. The TL1 and TL4 extracts were divided into two equal portions; one portion was used for the analysis of free amino acids, while the other was subjected to acid-vapor hydrolysis using $6 \mathrm{M} \mathrm{HCl}$ $\left(150{ }^{\circ} \mathrm{C}\right.$ for $3 \mathrm{~h}$; Glavin et al. 2006) to determine the total (free plus hydrolyzable) amino acid content. Only the total amino acid content (no "free" amino acid data) was determined for sample TL10a. All of the unhydrolyzed and acid-hydrolyzed extracts were redissolved in water, desalted using cation-exchange resins, and derivatized using OPA/NAC, following protocols described elsewhere (Glavin et al. 2006, 2011).

The derivatized amino acid content of the Tagish Lake samples and procedural blank was determined via UPLC-FD/ToF-MS using a Waters ACQUITY H Class UPLC with fluorescence detector and Waters Xevo G2 XS at NASA Goddard. The instrument parameters and analytical conditions used were similar to those described elsewhere (Glavin et al. 2006, 2011). For the Xevo mass calibrations, an automatically applied lock mass of a fragment (278.1141 Da) of Leucine Enkephalin $(0.09 \mu \mathrm{M}$ in $50 / 50$ acetonitrile/water with $0.1 \%$ formic acid) with a scan time of $1 \mathrm{~s}$ every $120 \mathrm{~s}$ was used. The capillary voltage was set to $1.2 \mathrm{kV}$. The amino acids and their enantiomeric ratios were quantified from the peak areas generated from both fluorescence detection and from the mass chromatogram of their OPA/NAC derivatives as described previously (Glavin et al. 2006). The reported amino acid abundances are the average value of three separate UPLC-FD/ToF-MS measurements. The errors given are based on the SD of the average value of three separate measurements. The TL1 and TL4 values are blankcorrected to account for trace levels of contamination present in the blanks. The TL10a abundances were not blank-corrected as the procedural blank contained unusually high levels of terrestrial amino acids that did not appear to similarly affect the TL10a sample.

Sufficient compound abundances allowed carbon isotopic compositions $\left(\delta^{13} \mathrm{C}\right)$ to be determined for glycine, $\beta$-alanine, and D- and L-alanine in both the unhydrolyzed and hydrolyzed TL1 extracts, and for $\mathrm{y}$-aminobutyric acid in the hydrolyzed extract. Nonhydrolyzed and hydrolyzed TL1 samples were dried down separately, as were procedural blanks. Each aliquot was dried under vacuum using a LabConco CentriVap centrifugal concentrator. Samples were esterified with isopropanol and the isopropyl esters reacted with trifluoroacetic anhydride (TFAA) using established methods (e.g., Elsila et al. 2009). The TFAA-isopropanol derivatives were dissolved in $5 \mu \mathrm{L}$ of ethyl acetate (Fisher Chemical, Optima Grade). The $\delta^{13} \mathrm{C}$ values of the TFAA-isopropanol derivatized samples were analyzed by gas chromatography coupled with mass spectrometry and isotope ratio mass spectrometry (GC-MS/IRMS), which provides compound-specific structural and stable isotopic information from a single sample injection. Details regarding the GC-MS/IRMS instrument, oven program, and data processing software are outlined in detail by Elsila et al. (2012). The final $\delta^{13} \mathrm{C}$ values of the amino acids in the samples and their precision were calculated as described elsewhere (Docherty et al. 2001; O'Brien et al. 2002; Elsila et al. 2009).

\section{Aldehydes and Ketones: Identification, Quantification, and $\delta^{13} \mathrm{C}$ Analysis}

Tagish Lake powders (see the Tagish Lake Samples and Procedural Blanks section) were stored and sealed in individual glass vials and kept cold $\left(<10^{\circ} \mathrm{C}\right)$ during shipment to the NASA Goddard Astrobiology Analytical Laboratory. At NASA Goddard, each sample was transferred to five $5 \mathrm{~mL}$ glass ampules in $\sim 0.5 \mathrm{~g}$ aliquots. Of water, $1 \mathrm{~mL}$ was added to each ampule, the ampules were flame-sealed and the solutions were heated at $100{ }^{\circ} \mathrm{C}$ for $24 \mathrm{~h}$. The water extracts were centrifuged and the supernatants were combined into one vial for each Tagish Lake specimen. The residual solids were rinsed three times each with $\sim 0.5 \mathrm{~mL}$ of water for each rinse. The supernatants were filtered through quartz wool to remove any solid remaining in suspension and the quartz wool filters were rinsed two times with $\sim 0.5 \mathrm{~mL}$ of water for each rinse. The combined water extracts and rinses resulted in final volumes of $\sim 13.5 \mathrm{~mL}$ for each specimen. The aldehydes and ketones in the water extracts were derivatized using an optimized EPA Method \#556 for PFBHA derivatization (Simkus et al. 2019) as follows: $1 \mathrm{~mL}$ of $0.2 \mathrm{mg} \mathrm{mL}^{-1}$ PFBHA solution was added to each water extract. The solutions were agitated for $5 \mathrm{~min}$ and left to react for $24 \mathrm{~h}$. One hundred microliters of $0.4 \mathrm{M}$ $\mathrm{HCl}$ solution was added to each solution to quench the reaction and $2 \mathrm{~mL}$ of $\mathrm{DCM}$ was added to each solution 
to extract the derivatized carbonyl compounds. The solutions were shaken for $5 \mathrm{~min}$ and then left undisturbed for $30 \mathrm{~min}$ to allow the DCM and water layers to separate and settle. The DCM layers were extracted and acid-washed ( $3 \mathrm{~mL}$ of $0.4 \mathrm{M} \mathrm{HCl})$. The DCM extraction of derivatized carbonyl compounds and subsequent acid-wash step was repeated for each specimen with another $2 \mathrm{~mL}$ of DCM. The $2 \times 2 \mathrm{~mL}$ DCM extracts containing the carbonyl derivatives were concentrated down to $200 \mu \mathrm{L}$ volumes under a stream of nitrogen and analyzed by GC-MS/IRMS.

The GC-MS analysis was carried out using a Thermo Trace GC equipped with a $5 \mathrm{~m}$ basedeactivated fused silica guard column (Restek, $0.25 \mathrm{~mm}$ ID) and three $30 \mathrm{~m}$ length $\times 0.25 \mathrm{~mm}$ I.D. $\times 0.5 \mu \mathrm{m}$ film thickness Rxi-5ms capillary columns (Restek) connected using Press-Tight connectors (Restek). The GC oven program was set as follows: initial temperature was $40{ }^{\circ} \mathrm{C}$, ramped at $10{ }^{\circ} \mathrm{C} \mathrm{min}-1$ to $160{ }^{\circ} \mathrm{C}$, ramped at $5^{\circ} \mathrm{C} \min ^{-1}$ to $190{ }^{\circ} \mathrm{C}$, ramped at $10{ }^{\circ} \mathrm{C} \min ^{-1}$ to $290{ }^{\circ} \mathrm{C}$, and held for $7 \mathrm{~min}$. The carrier gas used was ultrahigh purity helium (5.0 grade) at $2.6 \mathrm{~mL} \mathrm{~min}{ }^{-1}$ flow rate. Triplicate injections $(1 \mu \mathrm{L}$ aliquots) of the oxime derivative solutions were made in splitless mode. The mass spectrum was used to identify and quantify the individual meteoritic carbonyl compounds by comparison to reference standards and applying a calibration curve. A targeted ion mass-tocharge ratio $(m / z=181.0)$ was used to identify and quantify compounds. Approximately $10 \%$ of the sample eluting from the GC column was directed into a Thermo DSQII electron-impact quadrupole mass spectrometer (ion source set at $200{ }^{\circ} \mathrm{C}$ and $70 \mathrm{eV}$ ). The remaining $90 \%$ of each eluting compound was directed through a Thermo GC-C III interface for oxidation of the compounds to carbon dioxide. The carbon isotope analyses were then carried out on a Thermo MAT 253 IRMS. The $\delta^{13} \mathrm{C}$ values of the eluting compounds were obtained after injection of three pulses of precalibrated $\mathrm{CO}_{2} \quad\left(\delta^{13} \mathrm{C}=-24.23 \%\right.$ VPDB $)$ into the IRMS and computation using Thermo Isodat 2.5 software. To correct for the amount of carbon added by the derivatization reagent, underivatized standards were analyzed on a Costech ECS 4010 combustion elemental analyzer (EA) connected to the IRMS. The final $\delta^{13} \mathrm{C}$ values of the meteoritic aldehydes and ketones were calculated as described elsewhere (Docherty et al. 2001; Simkus et al. 2019).

\section{Monocarboxylic Acids: SPME and GC-MS}

Powdered samples of TL1, TL4, and TL10a (see the Tagish Lake Samples and Procedural Blanks section) were kept cold $\left(<0{ }^{\circ} \mathrm{C}\right)$ until their immediate transfer to
$5 \mathrm{~mL}$ glass ampules as $\sim 250 \mathrm{mg}$ aliquots. Of water, $2 \mathrm{~mL}$ was added to each ampule, the ampules were flame-sealed, and the samples were heated at $100{ }^{\circ} \mathrm{C}$ for $24 \mathrm{~h}$. The water extracts were transferred to centrifuge tubes and the residual solids were rinsed three times each with $\sim 0.5 \mathrm{~mL}$ of water for each rinse. After centrifugation, the supernatants were transferred to round-bottom flasks. The precipitates were rinsed three times each with $\sim 0.5 \mathrm{~mL}$ of water for each rinse. Following the procedure described by Hilts et al. (2014), the $\mathrm{pH}$ was adjusted to $\sim 11$ using $6 \mathrm{M} \mathrm{NaOH}$ to convert the carboxylic acids to nonvolatile carboxylate salts. The solutions were reduced to $<1 \mathrm{~mL}$ volumes using a rotary evaporator, and then the acids were regenerated by adjusting the $\mathrm{pH}$ to $\sim 2-3$ using $6 \mathrm{M}$ $\mathrm{HCl}$. The final sample volumes were measured and recorded and the solutions were analyzed via SPME technique. Gas chromatography separation of underivatized monocarboxylic acids was performed on an Agilent $6890 \mathrm{~N}$ using a $\mathrm{Nukol}^{\mathrm{TM}}$ column $(30 \mathrm{~m}$ length, $0.25 \mu \mathrm{m}$ film thickness, $0.25 \mathrm{~mm}$ diameter). The SPME fiber (carbowax-polyethylene glycol [PEG], 23gauge needle, $60 \mu \mathrm{m}$ thickness, $1 \mathrm{~cm}$ length fiber) was soaked in a sample solution for 10 min prior to manual $\mathrm{GC}$ injection. The GC injection port was held at $210{ }^{\circ} \mathrm{C}$ and injection of the SPME adsorbed samples was performed using splitless mode. The initial oven temperature was set at $35^{\circ} \mathrm{C}$ and held for $1 \mathrm{~min}$, after which the temperature was increased by $25^{\circ} \mathrm{C} \mathrm{min}-1$ to $135^{\circ} \mathrm{C}$, then $1.5^{\circ} \mathrm{C} \min ^{-1}$ to $185^{\circ} \mathrm{C}$, and then held at $185^{\circ} \mathrm{C}$ for $10 \mathrm{~min}$. Helium was the carrier gas at a constant flow rate of $1.0 \mathrm{~mL} \mathrm{~min}{ }^{-1}$. Detection was performed using an Agilent 5975C mass selective detector (MSD). Carboxylic acids were identified by comparing retention times and mass spectra with those of a suite of reference standards. Quantifications were determined by applying standard calibration curves for each individual compound. A trace amount of formic acid and acetic acid was present in the procedural blanks. The abundances of formic acid and acetic acid in TL1 and TL4 were blank-corrected by subtracting these abundances from the calculated concentrations. Concentrations were not determined for TL10a.

\section{DCM-Soluble Organic Compounds: GC-MS Analyses}

Powdered samples of TL1, TL4, and TL10a (see the Tagish Lake Samples and Procedural Blanks section) were kept cold until their transfer to $20 \mathrm{~mL}$ vials. Of DCM, $15 \mathrm{~mL}$ was added to each vial and the samples were stirred at room temperature for $24 \mathrm{~h}$ in order to extract weakly polar aliphatic and aromatic species. Following the extraction step, the DCM extracts were transferred to separate vials. The residual solid was 
rinsed three times with $0.5 \mathrm{~mL}$ of $\mathrm{DCM}$ for each rinse. The extracts were evaporated to $200 \mu \mathrm{L}$ volumes under a stream of $\mathrm{N}_{2}$ and then analyzed using GC-MS. Gas chromatography separation was performed on an Agilent $6890 \mathrm{~N}$ using a HP-5MS column (30 m length, $0.25 \mu \mathrm{m}$ film thickness, $250 \mu \mathrm{m}$ diameter) at MacEwan University. The initial oven temperature was $50{ }^{\circ} \mathrm{C}$ and held for $1 \mathrm{~min}$, after which the temperature was increased by $10{ }^{\circ} \mathrm{C} \mathrm{min}{ }^{-1}$ to a final temperature of $250{ }^{\circ} \mathrm{C}$. The temperature was held at $250{ }^{\circ} \mathrm{C}$ for $20 \mathrm{~min}$ for a total run time of $41 \mathrm{~min}$. Helium was the carrier gas at a constant flow rate of $1.0 \mathrm{~mL} \mathrm{~min}{ }^{-1}$. Samples were injected using pulsed splitless mode at $275^{\circ} \mathrm{C}$. Detection was performed using an Agilent 5975C MSD. Individual compounds were identified by analysis of the 2011 NIST Mass Spectral Library (version 2.0g).

\section{RESULTS}

\section{Amino Acid Distributions, $\delta^{13} \mathrm{C}$ Values, and Enantiomeric Compositions}

The absolute abundances of amino acids in the nonhydrolyzed extracts of TL1 and TL4 ("free" amino acids) and $6 \mathrm{M} \mathrm{HCl-hydrolyzed} \mathrm{extracts} \mathrm{of} \mathrm{TL1,} \mathrm{TL4,}$ and TL10a ("total" amino acids) are reported in Table 1. Examples of LC-FD chromatograms obtained for hydrolyzed extracts of the three Tagish Lake specimens and their corresponding blanks are shown in Fig. 1. TL1 was notably rich in amino acids ( $1874 \mathrm{ppb})$, while TL4 and TL10a were relatively depleted $(\sim 108$ and $\sim 139 \mathrm{ppb}$, respectively). Acid hydrolysis resulted in similar increases in the abundance of glycine for both HCl-hydrolyzed samples $(+133 \mathrm{ppb}$ for TL1 and $+72 \mathrm{ppb}$ for TL4). TL1 also showed a substantial increase in the abundance of $\gamma$-amino- $n$ butyric acid $(\gamma$-ABA) following acid hydrolysis (free $\gamma$ ABA abundance: $37.5 \mathrm{ppb}$; total $\gamma$-ABA abundance: $431.7 \mathrm{ppb})$. TL4 and TL10a contained very similar amino acid distributions, both dominated by glycine and containing relatively low levels of other amino acids, while the hydrolyzed extract of TL1 contained relatively high abundances of $\beta$-alanine and $\gamma$-ABA. Glycine, $\beta$-alanine, D,L-alanine, and $\gamma$-ABA were present in sufficient abundances in TL1 to obtain their compound-specific $\delta^{13} \mathrm{C}$ values (Table 2). The $\delta^{13} \mathrm{C}$ values for these amino acids span a wide range of positive values with $\gamma$-ABA exhibiting the lowest value $(+6 \%)$, followed by $\beta$-alanine $(+16 \%$ and $+22 \%)$. Glycine and alanine were similarly ${ }^{13} \mathrm{C}$-enriched, with values ranging between $+31 \%$ and $+47 \%$. The amino acid $\delta^{13} \mathrm{C}$ values from the nonhydrolyzed and hydrolyzed TL1 extracts fall within error of one another for all measurements. Table 3 lists the measured enantiomeric ratios $(\mathrm{D} / \mathrm{L})$ for amino acids detected in TL1, TL4, and TL10a. Isovaline and $\beta$-amino- $n$-butyric acid $(\beta-\mathrm{ABA})$ were near racemic $(\mathrm{D} / \mathrm{L}$ ratios $>0.87$ ) for all measurements. Glutamic acid exhibited an excess of the L-enantiomer for all measurements. Particularly low $\mathrm{D} / \mathrm{L}$ ratios were measured for serine in all samples, and for valine in TL10a. Despite near-racemic values for alanine, $\beta$-ABA, and isovaline, the TL10a amino acids generally exhibited the lowest $\mathrm{D} / \mathrm{L}$ ratios of the sample set (e.g., valine and aspartic acid).

\section{Aldehyde and Ketone Distributions and $\delta^{13} \mathrm{C}$ Compositions}

The sum total abundances of aldehydes and ketones steadily decrease from TL1 to TL10a to TL4 (Table 4). The three specimens contained similar suites of carbonyl compounds, with the exception of the relatively high abundance of acetaldehyde detected in TL1 and the absence of butyraldehyde and acetophenone in TL10a. Formaldehyde, acetaldehyde, and acetone were present in sufficient abundances in all three samples to obtain compound-specific $\quad \delta^{13} \mathrm{C}$ measurements (Table 4). Several of the carbonyl derivative peaks partially coeluted with either the unreacted PFBHA reagent or unknown organic compounds present in the samples. These partial co-elutions are noted in Table 4. The aldehyde and ketone $\delta^{13} \mathrm{C}$ values were ${ }^{13} \mathrm{C}$-depleted in comparison to the amino acid $\delta^{13} \mathrm{C}$ values. The formaldehyde $\delta^{13} \mathrm{C}$ values, ranging from $-51.5 \%$ to $-34.6 \%$, were exceptionally ${ }^{13} \mathrm{C}$-depleted. While the formaldehyde and acetone $\delta^{13} \mathrm{C}$ values were relatively consistent across the Tagish Lake specimens, the acetaldehyde $\delta^{13} \mathrm{C}$ values varied from $-23.3 \%$ to $-0.3 \%$, becoming progressively more ${ }^{13} \mathrm{C}$-enriched from TL4 to TL10a to TL1.

\section{Monocarboxylic Acid Distributions}

Monocarboxylic acids were detected in all three Tagish Lake specimens (Fig. 2). The most abundant acids in samples TL1 and TL4 were quantified by comparison to a series of reference standard curves (Table 5). Overall, TL1 is rich in monocarboxylic acids (>633 ppm) in comparison to TL4 (>474 ppm). Despite having similar concentrations for formic acid, TL1 and TL4 contain different relative abundances of the other carboxylic acids; TL1 contains relatively high abundances of acetic acid (225 ppm), propanoic acid (34 ppm), and trace amounts of higher molecular weight acids, while TL4 is relatively depleted in acetic acid (94 ppm) and lacks higher molecular weight compounds, aside from benzoic acid $(2.0 \mathrm{ppm})$ and a trace amount of octanoic acid. The TL1 and TL4 
Table 1. Amino acid abundances (ng $\mathrm{g}^{-1}$ ) from nonhydrolyzed ("free") and $6 \mathrm{M}$ HCl-hydrolyzed ("total") hot-water extracts.

\begin{tabular}{|c|c|c|c|c|c|}
\hline & \multicolumn{2}{|l|}{ TL1 } & \multicolumn{2}{|l|}{ TL4 } & \multirow{2}{*}{$\begin{array}{l}\text { TL10a } \\
\text { Total }^{\mathrm{a}}\end{array}$} \\
\hline & Free & Total & Free & Total & \\
\hline D-Aspartic acid & $6.23 \pm 0.50$ & $10.57 \pm 1.14$ & $0.42 \pm 0.02$ & $2.02 \pm 0.08$ & $2.14 \pm 0.09$ \\
\hline L-Aspartic acid & $6.86 \pm 0.51$ & $11.83 \pm 0.76$ & $0.46 \pm 0.04$ & $2.01 \pm 0.02$ & $4.07 \pm 0.04$ \\
\hline D-Glutamic acid & $0.89 \pm 0.20$ & $16.80 \pm 1.06$ & $<0.01$ & $0.80 \pm 0.10$ & $4.07 \pm 0.20$ \\
\hline L-Glutamic acid & $1.57 \pm 0.48$ & $22.28 \pm 0.53$ & $<0.01$ & $1.78 \pm 0.25$ & $7.14 \pm 1.20$ \\
\hline D-Serine & $1.14 \pm 0.25$ & $0.42 \pm 0.04$ & $<0.01$ & $0.04 \pm 0.01$ & $0.12 \pm 0.01$ \\
\hline L-Serine & $2.46 \pm 0.45$ & $4.24 \pm 2.04$ & $0.06 \pm 0.03$ & $<0.01$ & $0.83 \pm 0.06$ \\
\hline D-Threonine & $0.16 \pm 0.08$ & $<0.01$ & $<0.01$ & $0.36 \pm 0.15$ & $<0.01$ \\
\hline L-Threonine & $1.85 \pm 1.42$ & $1.13 \pm 0.24$ & $0.07 \pm 0.01$ & $0.10 \pm 0.04$ & $2.47 \pm 0.78$ \\
\hline Glycine & $426.35 \pm 41.58$ & $559.44 \pm 56.28$ & $20.28 \pm 1.63$ & $92.39 \pm 11.11$ & $85.21 \pm 7.03$ \\
\hline$\beta$-Alanine & $308.28 \pm 51.99$ & $371.19 \pm 66.05$ & $1.15 \pm 0.07$ & $2.32 \pm 0.10$ & $5.16 \pm 0.11$ \\
\hline D-Alanine & $66.16 \pm 9.95$ & $72.24 \pm 2.99$ & $0.78 \pm 0.02$ & $1.65 \pm 0.07$ & $4.77 \pm 0.46$ \\
\hline L-Alanine & $73.32 \pm 1.53$ & $72.22 \pm 2.67$ & $0.78 \pm 0.02$ & $2.12 \pm 0.06$ & $5.26 \pm 0.29$ \\
\hline$\gamma$-Amino- $n$-butyric acid $(\gamma$-ABA) & $37.45 \pm 2.67$ & $431.71 \pm 15.43$ & $0.10 \pm 0.04$ & $0.94 \pm 0.09$ & $6.12 \pm 0.27$ \\
\hline D- $\beta$-Amino- $n$-butyric acid (D- $\beta$-ABA) & $83.07 \pm 3.64$ & $63.00 \pm 5.08$ & $0.03 \pm 0.00$ & $0.05 \pm 0.01$ & $0.53 \pm 0.01$ \\
\hline L- $\beta$-Amino- $n$-butyric acid (L- $\beta$-ABA) & $87.36 \pm 2.89$ & $66.72 \pm 4.18$ & $0.04 \pm 0.00$ & $0.05 \pm 0.01$ & $0.53 \pm 0.00$ \\
\hline$\alpha$-Aminoisobutyric acid ( $\alpha$-AIB) & $16.25 \pm 0.87$ & $11.51 \pm 3.46$ & $0.26 \pm 0.04$ & $0.80 \pm 0.10$ & $4.90 \pm 0.06$ \\
\hline $\mathrm{D}, \mathrm{L}-\alpha-$-Amino- $n$-butyric acid $(\mathrm{D}, \mathrm{L}-\alpha-\mathrm{ABA})$ & $30.38 \pm 7.88$ & $35.86 \pm 6.00$ & $0.24 \pm 0.10$ & $<0.01$ & $1.44 \pm 0.54$ \\
\hline$\varepsilon$-Amino- $n$-caproic acid (EACA) & $8.05 \pm 0.66$ & $23.09 \pm 2.09$ & $<0.01$ & $0.16 \pm 0.04$ & $3.19 \pm 0.28$ \\
\hline $\begin{array}{l}\text { 3-Amino-2,2-dimethylpropanoic } \\
\text { acid (3-a-2,2-dmpa) }\end{array}$ & $1.72 \pm 0.07$ & $4.48 \pm 0.17$ & $0.01 \pm 0.00$ & $<0.01$ & $<0.01$ \\
\hline D,L-4-Aminopentanoic acid (D,L-4-apa) & $5.28 \pm 0.38$ & $27.10 \pm 1.21$ & $<0.01$ & $<0.01$ & $<0.01$ \\
\hline $\begin{array}{l}\text { D,L-4-Amino-3-methylbutanoic } \\
\text { acid (D,L-4-a-3-mba) }\end{array}$ & $0.14 \pm 0.01$ & $2.41 \pm 0.19$ & $<0.01$ & $<0.01$ & $<0.01$ \\
\hline $\begin{array}{l}\text { D,L-3-amino-2-methylbutanoic } \\
\text { acid (D,L-3-a-2-mba) }\end{array}$ & $4.39 \pm 0.95$ & $1.95 \pm 0.12$ & $<0.01$ & $<0.01$ & $<0.01$ \\
\hline $\begin{array}{l}\text { D,L-3-Amino-2-ethylpropanoic } \\
\text { acid (D,L-3-a-2-epa) }\end{array}$ & $1.49 \pm 0.15$ & $1.38 \pm 0.16$ & $<0.01$ & $<0.01$ & $<0.01$ \\
\hline 5-Aminopentanoic acid (5-apa) & $1.51 \pm 0.18$ & $13.38 \pm 1.19$ & $<0.01$ & $<0.01$ & $<0.01$ \\
\hline $\begin{array}{l}\text { D,L-4-Amino-2-methylbutanoic } \\
\text { acid (D,L-4-a-2-mba) }\end{array}$ & $1.38 \pm 0.18$ & $35.73 \pm 2.07$ & $<0.01$ & $<0.01$ & $<0.01$ \\
\hline 3-Amino-3-methylbutanoic acid (3-a-3-mba) & $<0.01$ & $<0.01$ & $<0.01$ & $<0.01$ & $<0.01$ \\
\hline D-2-Amino-2-methylbutanoic acid (D-isovaline) & $0.21 \pm 0.02$ & $<0.01^{\mathrm{b}}$ & $<0.01$ & $<0.01$ & $0.27 \pm 0.01$ \\
\hline L-2-Amino-2-methylbutanoic acid (L-isovaline) & $0.24 \pm 0.02$ & $<0.01^{\mathrm{b}}$ & $<0.01$ & $<0.01$ & $0.27 \pm 0.02$ \\
\hline D,L-3-aminopentanoic acid (D,L-3-apa) & $5.81 \pm 0.34$ & $5.43 \pm 0.36$ & $<0.01$ & $<0.01$ & $<0.01$ \\
\hline D-2-Amino-3-methylbutanoic acid (D-valine) & $0.47 \pm 0.11$ & $1.28 \pm 0.08$ & $<0.01$ & $<0.01$ & $0.04 \pm 0.00$ \\
\hline L-2-Amino-3-methylbutanoic acid (L-valine) & $0.62 \pm 0.08$ & $1.23 \pm 0.05$ & $<0.01$ & $0.43 \pm 0.20$ & $0.69 \pm 0.03$ \\
\hline D-2-Aminopentanoic acid (D-norvaline) & $<0.01$ & $0.11 \pm 0.05$ & $<0.01$ & $<0.01$ & $<0.01$ \\
\hline L-2-Aminopentanoic acid (L-norvaline) & $<0.01$ & $0.12 \pm 0.05$ & $<0.01$ & $<0.01$ & $<0.01$ \\
\hline Total abundance & 1181.1 & 1873.9 & 23.8 & 108.0 & 139.2 \\
\hline
\end{tabular}

${ }^{\mathrm{a}}$ TL10a abundances are not blank-corrected values.

${ }^{\mathrm{b}}$ Hydrolyzed TL1 contained trace amounts of D- and L-isovaline that could not be quantified as they co-eluted with a relatively large D,L-3-apa peak.

abundances for formic and acetic acid have been corrected to account for the trace amounts of formic acid $(0.6 \mu \mathrm{mol})$ and acetic acid $(1.5 \mu \mathrm{mol})$ present in the mineral analog blank. Unlike TL1 and TL4, the monocarboxylic acids in the TL10a sample appeared to be primarily terrestrial in origin; the only monocarboxylic acids detected in TL10a were acetic, hexanoic, heptanoic, and octanoic acid-all compounds present in the mineral analog blank (Fig. 2). Thus, monocarboxylic acids were not quantified in TL10a and were excluded from further discussions throughout this work. Benzoic acid appears to be intrinsic to TL10a as it was not present in the procedural blank.

\section{DCM-Soluble Organic Compounds}

The GC-MS chromatograms for the DCM extracts of samples TL1, TL4, and TL10a are 


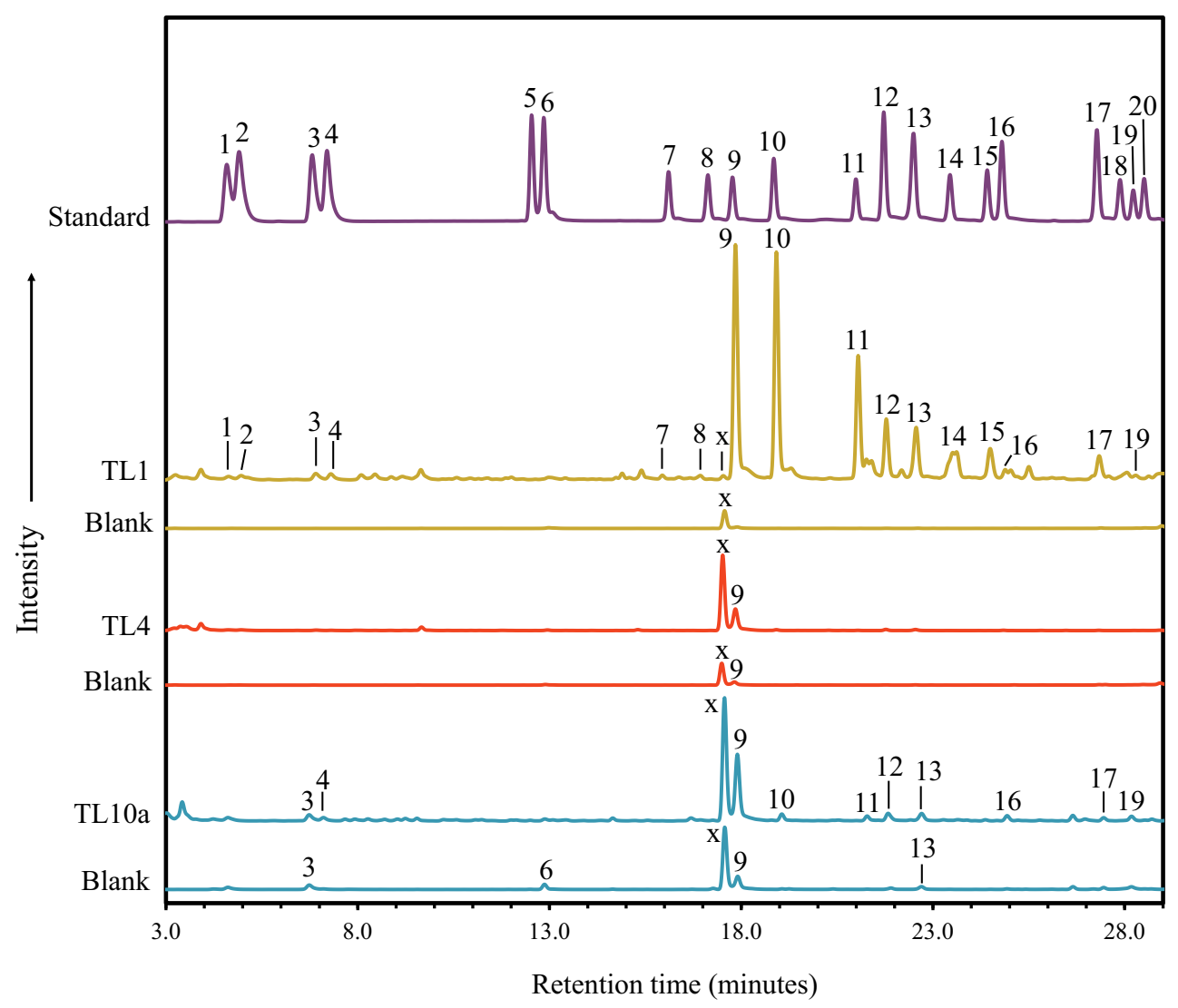

Fig. 1. The 3-29 min region of LC-FD chromatograms for OPA/NAC amino acid analyses of 6 M HCl-hydrolyzed hot-water extracts of TL1, TL4, TL10a, and their corresponding procedural blanks, compared to a standard amino acid mixture. Peak identifications (1) D-aspartic acid, (2) L-aspartic acid, (3) L-glutamic acid, (4) D-glutamic acid, (5) D-serine, (6) L-serine, (7) D-threonine, (8) L-threonine, (9) glycine, (10) $\beta$-alanine, (11) $\gamma$-amino- $n$-butyric acid, (12) D-alanine, (13) L-alanine, (14) D- $\beta$ amino-n-butyric acid, (15) L- $\beta$-amino- $n$-butyric acid, (16) $\alpha$-aminoisobutyric acid, (17) D,L- $\alpha$-amino-n-butyric acid, (18) Disovaline, (19) $\varepsilon$-amino- $n$-caproic acid (EACA), (20) L-isovaline. An unidentified fluorescent desalting artifact is labeled with an $\mathrm{X}$. Amino acid abundances are reported in Table 1 .

Table 2. Compound-specific carbon isotopic measurements (\% VPDB) of amino acids from the nonhydrolyzed ("free") and $6 \mathrm{M} \mathrm{HCl}$-hydrolyzed ("total") hot-water extracts of Tagish Lake specimen TL1, compared to the previously analyzed specimens TL5b and TL11h (data from Glavin et al. 2012).

\begin{tabular}{|c|c|c|c|c|}
\hline \multirow{2}{*}{$\begin{array}{l}\text { Amino } \\
\text { acid }\end{array}$} & \multicolumn{2}{|l|}{ TL1 } & \multirow{2}{*}{$\begin{array}{l}\text { TL5b Total, } \\
\text { Glavin } \\
\text { et al. } \\
(2012)\end{array}$} & \multirow{2}{*}{$\begin{array}{l}\text { TL11h } \\
\text { Total, } \\
\text { Glavin } \\
\text { et al. (2012) }\end{array}$} \\
\hline & Free & Total & & \\
\hline Glycine & $+31 \pm 5$ & $+39 \pm 7$ & $+39 \pm 6$ & $+19 \pm 4$ \\
\hline$\beta$-Alanine & $+22 \pm 6^{\mathrm{a}}$ & $+16 \pm 5^{a}$ & $+30 \pm 6$ & $-5 \pm 4$ \\
\hline D-Alanine & $+37 \pm 3$ & $+34 \pm 13$ & $+67 \pm 7$ & $+6 \pm 3$ \\
\hline L-Alanine & $+42 \pm 7$ & $+47 \pm 4$ & $+55 \pm 3$ & $+16 \pm 4$ \\
\hline$\gamma-\mathrm{ABA}$ & n.d. & $+6 \pm 5$ & n.d. & $+4 \pm 3$ \\
\hline
\end{tabular}

n.d. $=$ not determined.

${ }^{\mathrm{a}} \beta$-alanine contains some co-eluting D,L- $\beta$-ABA. illustrated in Fig. 3. Table 6 lists the weakly polar aliphatic and aromatic species identified in the extracts by reference to the NIST Mass Spectral Library. Confidence values reported by the NIST Library for compound identifications ranged from $<20 \%$ to $60 \%$ for aliphatic and monoaromatic compounds and from $35 \%$ to $60 \%$ for polycyclic aromatic hydrocarbons (PAHs). The compositions of TL1 and TL4 are nearly identical in terms of similar abundances of weakly polar aliphatic hydrocarbons. The NIST Mass Spectral Library identified some PAHs (naphthalene, fluoranthene, pyrene) which were absent in both the TL4 and TL10a samples. Peaks 1, 2, 6, 7, 18, and 21 (identified by the NIST Mass Spectral Library as 3,5,5trimethyl-2-hexene, 1,2,3,4,5-pentamethyl-cyclopentane, 2,4,4-trimethyl-1-hexene, undecane, and dodecane) in the TL1 and TL4 samples were also present in similar 
Table 3. Amino acid enantiomeric ratio (D/L) measured in the nonhydrolyzed ("free") and $6 \mathrm{M}$ HCl-hydrolyzed ("total") hot-water extracts of TL1, TL4, and TL10a.

\begin{tabular}{|c|c|c|c|c|c|}
\hline \multirow[b]{2}{*}{ Amino acid } & \multicolumn{2}{|l|}{ TL1 } & \multicolumn{2}{|l|}{ TL4 } & \multirow{2}{*}{$\begin{array}{l}\text { TL10a } \\
\text { Total }\end{array}$} \\
\hline & Free & Total & Free & Total & \\
\hline Aspartic acid & $0.91 \pm 0.10$ & $0.89 \pm 0.11$ & $0.93 \pm 0.09$ & $1.00 \pm 0.04$ & $0.53 \pm 0.02$ \\
\hline Glutamic acid & $0.57 \pm 0.22$ & $0.75 \pm 0.05$ & n.d. & $0.45 \pm 0.08$ & $0.57 \pm 0.10$ \\
\hline Serine & $0.46 \pm 0.13$ & $0.10 \pm 0.05$ & $0.10 \pm 0.07$ & n.d. & $0.14 \pm 0.01$ \\
\hline Alanine & $0.90 \pm 0.14$ & $0.94 \pm 0.05$ & $1.00 \pm 0.03$ & $0.78 \pm 0.04$ & $0.91 \pm 0.10$ \\
\hline$\beta-A B A$ & $0.95 \pm 0.05$ & $0.94 \pm 0.10$ & $0.96 \pm 0.05$ & $0.93 \pm 0.11$ & $1.00 \pm 0.03$ \\
\hline Isovaline & $0.87 \pm 0.12$ & n.d. & n.d. & n.d. & $1.00 \pm 0.07$ \\
\hline Valine & $0.76 \pm 0.20$ & $1.03 \pm 0.08$ & n.d. & n.d. & $0.06 \pm 0.01$ \\
\hline
\end{tabular}

n.d. $=$ not determined.

Nonprotein amino acids are shown in italics.

Table 4. Concentrations ( $\left.\mathrm{ng} \mathrm{g}^{-1}\right)$ and compound-specific $\delta^{13} \mathrm{C}$ compositions (\%o VPDB) for carbonyl compounds in hot-water extracts of samples TL1, TL4, and TL10a.

\begin{tabular}{|c|c|c|c|c|c|c|}
\hline \multirow[b]{2}{*}{ Compound } & \multicolumn{2}{|l|}{ TL1 } & \multicolumn{2}{|l|}{ TL4 } & \multicolumn{2}{|l|}{ TL10a } \\
\hline & $\begin{array}{l}\text { Abundance }^{\mathrm{a}} \\
(\mathrm{n} / \mathrm{g})\end{array}$ & $\delta^{13} \mathrm{C}(\%)$ & $\begin{array}{l}\text { Abundance }{ }^{\mathrm{a}} \\
(\mathrm{n} / \mathrm{g})\end{array}$ & $\delta^{13} \mathrm{C}(\%)$ & Abundance $^{\mathrm{a}}(\mathrm{n} / \mathrm{g})$ & $\delta^{13} \mathrm{C}(\%)$ \\
\hline \multicolumn{7}{|l|}{ Aldehydes } \\
\hline Formaldehyde & $419.2 \pm 18.7$ & $-40.7^{b} \pm 4.2$ & $96.7 \pm 3.7$ & $-51.5^{\mathrm{b}} \pm 7.9$ & $664.5 \pm 28.6$ & $-34.6^{\mathrm{b}} \pm 2.5$ \\
\hline Acetaldehyde & $2474.1 \pm 57.1$ & $\begin{array}{l}(E):-0.3 \pm 1.4 \\
(Z):-3.9 \pm 2.0\end{array}$ & $84.1 \pm 1.0$ & $\begin{array}{l}(E):-23.3^{\mathrm{c}} \pm 6.7 \\
(Z): \text { n.d. }\end{array}$ & $953.0 \pm 17.0$ & $\begin{array}{l}(E):-12.3 \pm 0.7 \\
(Z):-12.2 \pm 0.9\end{array}$ \\
\hline Propionaldehyde & $217.4 \pm 4.5$ & n.d. & $34.2 \pm 0.5$ & n.d. & $73.3^{\mathrm{a}} \pm 0.4$ & n.d. \\
\hline Butyraldehyde & $134.7 \pm 1.2$ & n.d. & $48.4 \pm 1.8$ & n.d. & n.d. & n.d. \\
\hline \multicolumn{7}{|l|}{ Ketones } \\
\hline Acetone & $616.0 \pm 187.1$ & $-13.4^{\mathrm{b}} \pm 1.9$ & $44.9 \pm 1.8$ & $-19.1^{b} \pm 7.3$ & $184.3 \pm 18.0$ & $-12.8^{\mathrm{b}} \pm 2.4$ \\
\hline 2-Butanone & $154.4 \pm 40.3$ & n.d. & $33.7^{\mathrm{d}} \pm 1.1$ & n.d. & $61.8^{\mathrm{d}} \pm 1.3$ & n.d. \\
\hline Acetophenone & $6.6^{\mathrm{e}} \pm 0.9$ & n.d. & $3.9^{\mathrm{e}} \pm 0.6$ & n.d. & n.d. & n.d. \\
\hline Total abundance & $4022.3 \pm 285.7$ & & $345.93 \pm 4.94$ & & $1936.9 \pm 64.8$ & \\
\hline
\end{tabular}

n.d. $=$ not determined.

${ }^{\mathrm{a}}$ Abundances represent the sum of the $(E)$ and $(Z)$ isomers where applicable, unless otherwise noted.

${ }^{\mathrm{b}}$ Partially co-eluting with an unknown compound.

${ }^{\mathrm{c}}$ Partially co-eluting with unreacted PFBHA reagent.

${ }^{\mathrm{d}}$ Abundances for 2-butanone in TL4 and TL10a represent the $(Z)$ isomer only. (E)-2-butanone peaks were below the quantification limit.

${ }^{\mathrm{e}} \mathrm{Abundances}$ for acetophenone represent the $(Z)$ isomer only. $(E)$-acetophenone peaks were below the quantification limit.

abundances in the corresponding procedural blanks and are, therefore, presumed to be terrestrial contaminants. The TL10a sample appeared to be devoid of aliphatic and aromatic compounds; the DCM extraction only yielded a large elemental sulfur $\left(\mathrm{S}_{8}\right)$ peak and two small peaks identified by the NIST Mass Spectral Library as 4-methyl-2-pentanol and 2ethyl-1-hexanol. These two peaks were observed in similar trace abundances in the corresponding procedural blanks and are assumed to be terrestrial in origin. Consistent with these results, a similar DCM extraction on a separate subsample of TL10a (0.414 g) yielded no detectable aliphatic or aromatic organic compounds. Based on the GC-MS peak areas of $\mathrm{S}_{8}$ (eluting at $\sim 19.4 \mathrm{~min}$ ), TL1 and TL10a yielded similar abundances of dissolved sulfur $\left(\mathrm{S}_{8}\right)$, while TL4 yielded a notably smaller abundance.

\section{DISCUSSION}

\section{General Observations and Total Soluble Organic Contents}

The Tagish Lake specimens TL1, TL4, and TL10a were selected for this study based on macroscopic differences observed in hand-sample (i.e., presence or absence of chondrules, grain size, etc.), with the aim to cover the full range of lithological and chemical compositions contained within the Tagish Lake meteorite. In terms of macroscopic appearances, 

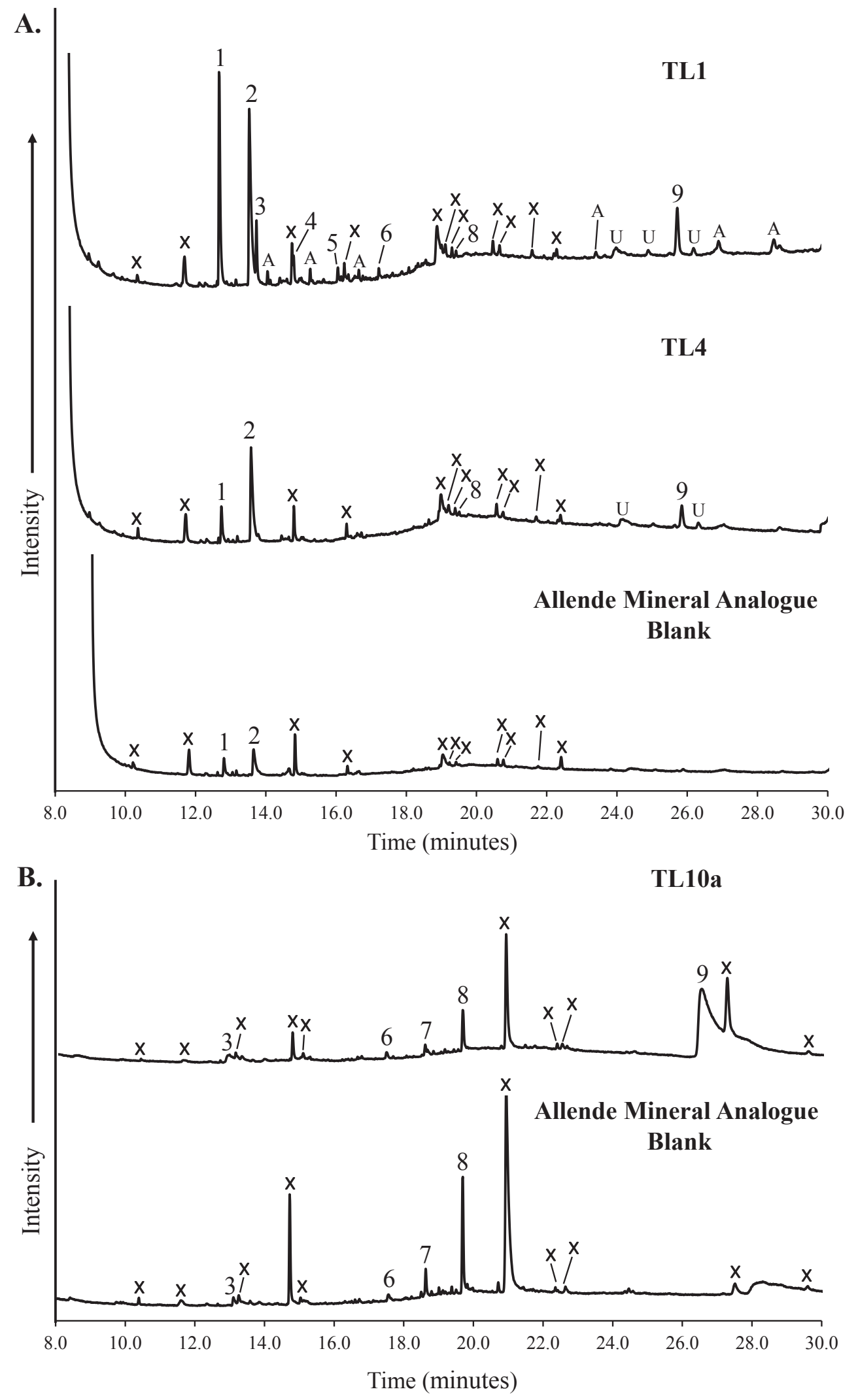
Fig. 2. GC-MS chromatograms for SPME analyses of hot-water extracts of (A) TL1, TL4, and the corresponding Allende mineral analog blank; and (B) TL10a and the corresponding Allende mineral analog blank. Water extractions were carried out on similar masses for the three specimens: $0.490 \mathrm{~g}$ of TL1, $0.500 \mathrm{~g}$ of TL4, and $0.640 \mathrm{~g}$ of TL10a. The final sample volumes are: $1.56 \mathrm{~mL}$ for TL1, $2.30 \mathrm{~mL}$ for TL4, and $1.52 \mathrm{~mL}$ for TL10a. All analyses were carried out using the same laboratory procedure. Any observed differences in composition between the procedural blanks are attributed to temporal variations of background levels of organics within the laboratory and/or reagents. Peak identifications (1) acetic acid, (2) formic acid, (3) propanoic acid, (4) butanoic acid, (5) pentanoic acid, (6) hexanoic acid, (7) heptanoic acid, (8) octanoic acid, (9) benzoic acid, (X) reagent or contaminant present in the sample and corresponding blank, (A) aliphatic carboxylic acid not present in corresponding blank, (U) unknown compound not present in corresponding blank. Peaks representing formic, acetic, propanoic, hexanoic, heptanoic, octanoic, and benzoic acid were identified by comparison to retention times and mass spectra of carboxylic acid reference standards. Unknown aliphatic carboxylic acids (A) were identified based on best matches reported by the NIST Mass Spectral Library.

samples TL1 and TL10a appear similar to TL5b and were, therefore, originally both priority samples for targeting relatively high abundances of organic compounds. In comparison to the TL1 and TL10a lithologies, the TL4 specimen was relatively indurated and difficult to subsample and powder, consistent with the observation of a relatively low abundance of soft phyllosilicate minerals (saponite and serpentine clays) and amorphous material (Gilmour et al. 2019). TL4 also has a uniquely high porosity (36\% versus $30 \%$ for the rest of the sample suite; Ralchenko et al. 2014), suggesting that the primary pore spaces have remained devoid of secondary sheet silicates (Blinova et al. 2014a). The relatively low abundance of secondary minerals suggests that TL4 has experienced a relatively low degree of aqueous alteration compared to the other two specimens (Gilmour et al. 2019). Concurrent hot-water extractions of TL1, TL4, and TL10a revealed another notable difference in their compositions, as evidenced by varying amounts of a dark, water-insoluble film in the extracts (Fig. 4); larger amounts of the oily film in the TL1 extract suggests that the specimen has a relatively higher water-insoluble organic content.

The soluble organic data for the three newly analyzed Tagish Lake specimens can be described as two general groupings based on total abundances of organics, i.e., (1) sample TL1, containing a relatively high overall soluble organic content and sharing compositional similarities with the previously analyzed Tagish Lake lithologies TL5b and TL11h (Herd et al. 2011; Glavin et al. 2012; Hilts et al. 2014), and (2) samples TL4 and TL10a, which were found to be generally depleted in soluble organic compounds. The composition of TL1 appears to reflect the previously described "sweet spot" of aqueous alteration, whereby an intermediate level or extent of alteration allows for synthesis and preservation of large abundances of soluble organics, while the lower and higher endmembers of the alteration sequence either result in poor production or poor preservation of the compounds (Herd et al. 2011). The sum total of amino acids $(\sim 1874 \mathrm{ppb})$ and carboxylic acids $(\sim 634 \mathrm{ppm})$ in TL1 is intermediate between those of the previously
Table 5. Monocarboxylic acid abundances (ppm) of TL1 and TL4 (this study) compared to abundances (ppm) for TL5b, TL11h, TL11i, TL11v reported by Hilts et al. (2014).

\begin{tabular}{|c|c|c|c|c|c|c|}
\hline & \multicolumn{2}{|c|}{ This study } & \multicolumn{4}{|c|}{ Hilts et al. (2014) } \\
\hline & TL1 & TL4 & TL5b & TL11h & TL11i & TL11v \\
\hline Formic & 368 & 378 & 209 & 340 & 260 & 309 \\
\hline Acetic & 225 & 94 & 293 & 240 & 92 & 71 \\
\hline Propanoic & 34 & n.d. & 9 & 57 & 4 & 5 \\
\hline Butanoic & n.q. ${ }^{a}$ & n.d. & 7 & 13 & 1 & 1 \\
\hline Pentanoic & n.q. & n.d. & n.q. & n.d. & n.d. & n.d. \\
\hline Hexanoic & 2.8 & n.d. & n.q. & 3 & 0.9 & 0.6 \\
\hline Octanoic & 1.7 & n.q. & 2 & 2 & 0.2 & 0.1 \\
\hline Benzoic & 2.0 & 2.4 & n.q. & n.q. & n.q. & n.q. \\
\hline Total $^{\mathrm{b}}$ & 633.5 & 474.4 & 533 & 656 & 359 & 387 \\
\hline
\end{tabular}

n.q. = not quantified; compound was detected but not quantifiable. n.d. $=$ not detected.

${ }^{\mathrm{a}}$ Butanoic acid co-eluted with an unknown contaminant.

${ }^{\mathrm{b}}$ Total abundances for TL5b, TL11h, TL11i, and TL11v include monocarboxylic acids that were not identified in TL1 and TL4 (not listed here).

analyzed TL5b and TL11h samples, which have been discussed as having experienced moderate levels of alteration (Herd et al. 2011; Glavin et al. 2012; Hilts et al. 2014). Although TL4 and TL10a resemble the previously analyzed TL11i specimen in terms of low total abundances of organic compounds, there are several notable differences in their compositions, potentially indicating unique alteration histories. Here, we discuss in detail the distribution of a range of compound classes in Tagish Lake, aiming to elucidate formation mechanisms and to gain insight into which conditions were most influential in yielding relatively high abundances of organics in TL1.

\section{Amino Acid Synthesis and the Influence of Aqueous Alteration}

The amino acid content of the Tagish Lake meteorite has been shown to vary widely across the 

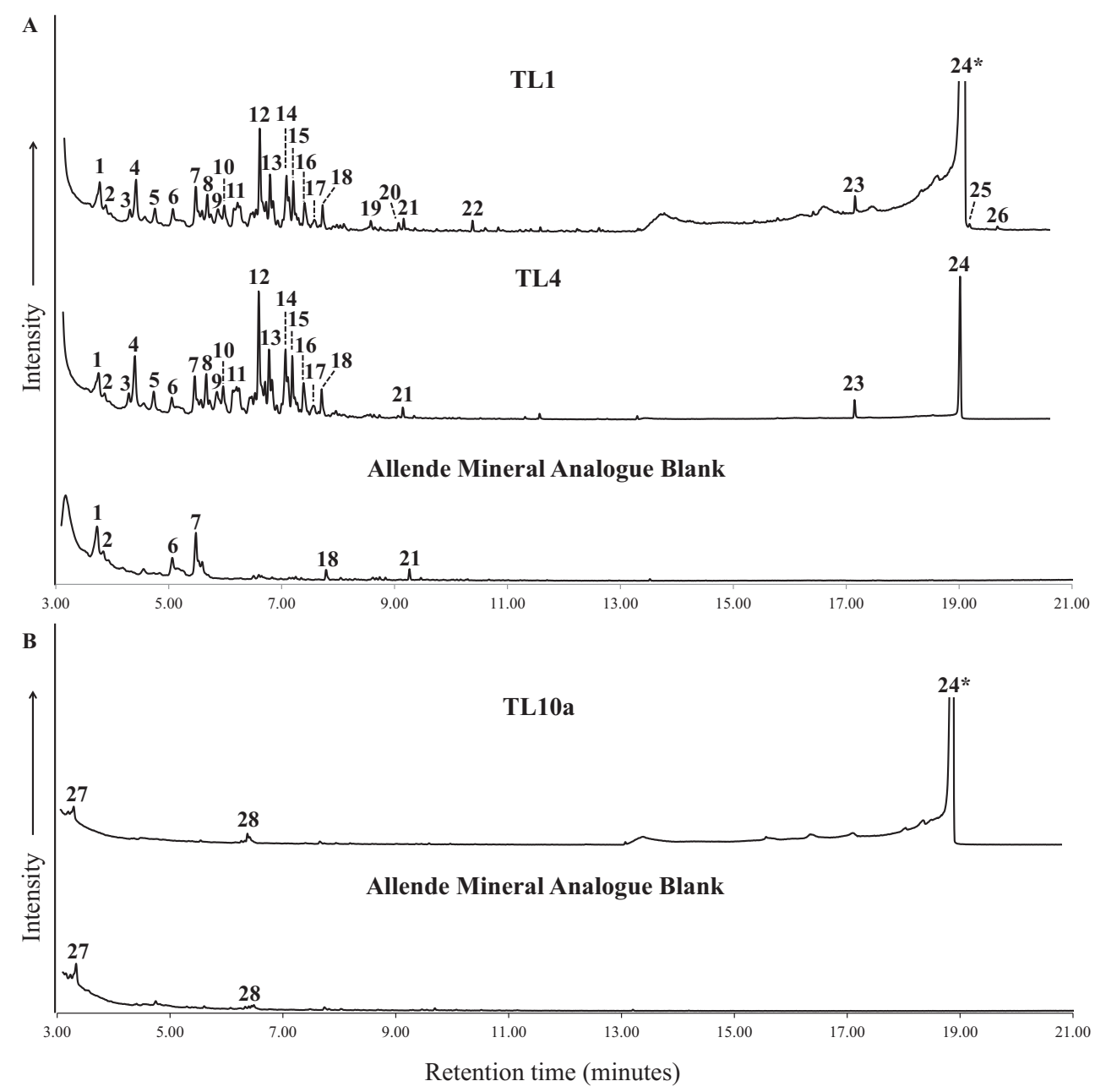

Fig. 3. Stacked GC-MS chromatograms for DCM extracts ( $1 \mu \mathrm{L}$ injections from $200 \mu \mathrm{L}$ volumes) of Tagish lake samples (A) TL1, TL4, and the corresponding Allende mineral analog blank; and (B) TL10a and the corresponding Allende mineral analog blank. The cyclic octaatomic sulfur peaks ${ }^{(*)}$ in samples TL1 and TL10a have been truncated. Compound identifications are listed in Table 6.

range of lithologies, not only in terms of total abundances but also in terms of the amino acid distributions and their compound-specific carbon isotope compositions (Pizzarello et al. 2001; Herd et al. 2011; Glavin et al. 2012; Hilts et al. 2014). The molar relative abundances (glycine $=1.0$ ) of the common meteoritic amino acids $\gamma$-ABA, $\alpha$-alanine, $\beta$-alanine, $\alpha$ AIB, and isovaline (Fig. 5) illustrate this variability across Tagish Lake specimens. The amino acid composition of TL1 is unique in that it contains particularly high absolute abundances of $\beta$-alanine and $\gamma$-ABA (Table 1); TL1 has the highest reported abundances for these two compounds in Tagish Lake so far. The bulk of the $\gamma$-ABA in TL1 was observed only in the acid-hydrolyzed sample, suggesting that the compound was present as a labile amino acid polymer that was converted to $\gamma$-ABA upon acid hydrolysis
(Cronin 1976; Glavin et al. 2011). Unlike TL5b and TL11h, isovaline and $\alpha$-AIB, common $\alpha$-amino acids in CM chondrites, are present in only trace amounts in TL1. A similar amino acid composition has been observed for the CI chondrites Orgueil and Ivuna (Ehrenfreund et al. 2001); their relatively high abundances of $\beta$-alanine and $\gamma$-ABA and relatively low abundances of $\alpha$-amino acids were attributed to amino acid synthesis in low-temperature conditions and/or in the absence of Strecker $\alpha$-amino acid precursors (e.g., $\mathrm{HCN})$. The latter scenario is consistent with the relatively high abundance of aldehydes and ketones observed in TL1, as aldehydes and ketones could have remained unreacted if the other Strecker precursors (e.g., HCN) were limiting in the system. The collective data suggest that Strecker synthesis was not the dominant reaction involved in amino acid production in 
Table 6. Weakly polar aliphatic and aromatic compounds and $\mathrm{S}_{8}$ detected in DCM extracts of samples TL1, TL4, and TL10a. Compound identifications are best matches reported by the NIST Mass Spectral Library.

\begin{tabular}{|c|c|c|c|c|c|}
\hline Compound & Peak & Compound & Peak & Compound & Peak \\
\hline Sample TL1 & & Sample TL4 & & Sample TL10a & \\
\hline 3,5,5-Trimethyl-2-hexene ${ }^{\mathrm{a}}$ & 1 & 3,5,5-Trimethyl-2-hexene ${ }^{\mathrm{a}}$ & 1 & 4-methyl-2-pentanol ${ }^{\mathrm{a}}$ & 27 \\
\hline 3,5,5-Trimethyl-2-hexene $e^{\mathrm{a}, \mathrm{b}}$ & 2 & 3,5,5-Trimethyl-2-hexene $e^{\mathrm{a}, \mathrm{b}}$ & 2 & 2-ethyl-1-hexanol ${ }^{\mathrm{a}}$ & 28 \\
\hline Ethylbenzene & 3 & Ethylbenzene & 3 & $\mathrm{~S}_{8}$ & 24 \\
\hline 1,3-Dimethyl-benzene & 4 & 1,3-Dimethyl-benzene & 4 & & \\
\hline p-Xylene & 5 & p-Xylene & 5 & & \\
\hline $1,2,3,4,5$-Pentamethyl-cyclopentane $\mathrm{a}^{\mathrm{a}}$ & 6 & $1,2,3,4,5$-Pentamethyl-cyclopentane $\mathrm{a}^{\mathrm{a}}$ & 6 & & \\
\hline 2,4,4-Trimethyl-1-hexene ${ }^{\mathrm{a}}$ & 7 & 2,4,4-Trimethyl-1-hexene ${ }^{\mathrm{a}}$ & 7 & & \\
\hline 2,6-Dimethyl-undecane ${ }^{\mathrm{b}}$ & 8 & 2,2,6-Trimethyl-octane ${ }^{\mathrm{b}}$ & 8 & & \\
\hline 2,2,6-Trimethyl-octane & 9 & 2,2,6-Trimethyl-octane & 9 & & \\
\hline 2,2,6-Trimethyl-octane ${ }^{\mathrm{b}}$ & 10 & 2,2,6-Trimethyl-octane $\mathrm{e}^{\mathrm{b}}$ & 10 & & \\
\hline Mesistylene & 11 & Mesistylene & 11 & & \\
\hline 2,2-Dimethyl-decane & 12 & 2,2-Dimethyl-decane & 12 & & \\
\hline 6-Ethyl-2-methyl-octane ${ }^{\mathrm{b}}$ & 13 & 2,3,6,7-Tetramethyl-octane ${ }^{\mathrm{b}}$ & 13 & & \\
\hline 5-Ethyl-2,2,3-trimethyl-heptane $\mathrm{b}^{\mathrm{b}}$ & 14 & 3-Methyl-nonane ${ }^{\mathrm{b}}$ & 14 & & \\
\hline 2,6-Dimethyl-octane ${ }^{\mathrm{b}}$ & 15 & 3,6-Dimethyl-undecane ${ }^{\mathrm{b}}$ & 15 & & \\
\hline 3-Methyl-undecane ${ }^{\mathrm{b}}$ & 16 & 2,6,10-Trimethyl-dodecane ${ }^{\mathrm{b}}$ & 16 & & \\
\hline 3,7-Dimethyl-decane ${ }^{\mathrm{b}}$ & 17 & 4-Methyl-dodecane ${ }^{\mathrm{b}}$ & 17 & & \\
\hline Undecane $^{\mathrm{a}}$ & 18 & Undecane $^{\mathrm{a}, \mathrm{b}}$ & 18 & & \\
\hline 1,2,4,5-Tetramethyl-benzene ${ }^{\mathrm{b}}$ & 19 & Dodecane $^{\mathrm{a}}$ & 21 & & \\
\hline Naphthalene & 20 & $\begin{array}{l}\text { 1,2-Benzene-dicarboxylic acid, } \\
\text { bis(2-methylpropyl) ester }{ }^{\mathrm{b}}\end{array}$ & 23 & & \\
\hline Dodecane $^{\mathrm{a}}$ & 21 & $\mathrm{~S}_{8}$ & 24 & & \\
\hline Pentamethyl-benzene & 22 & & & & \\
\hline $\begin{array}{l}\text { 1,2-benzene-dicarboxylic acid, } \\
\text { bis(2-methylpropyl) ester }{ }^{\mathrm{b}}\end{array}$ & 23 & & & & \\
\hline $\mathrm{S}_{8}$ & 24 & & & & \\
\hline Fluoranthene & 25 & & & & \\
\hline Pyrene & 26 & & & & \\
\hline
\end{tabular}

${ }^{\mathrm{a}}$ Terrestrial contaminant.

${ }^{\mathrm{b}}$ Confidence level reported by the NIST Mass Spectral Library $<20 \%$ probability.

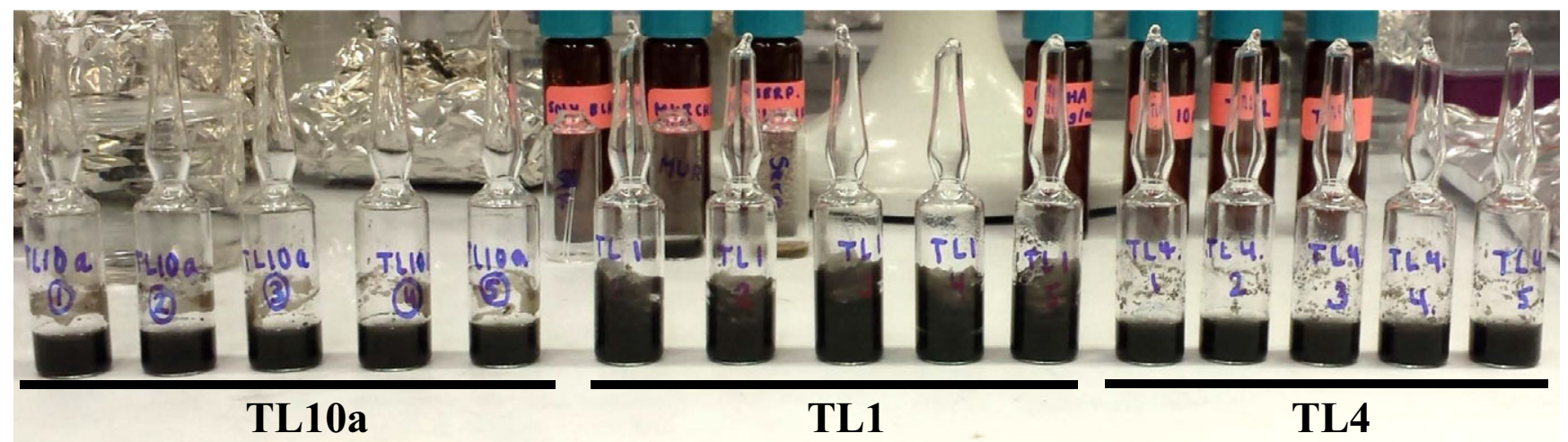

Fig. 4. Flame-sealed glass ampules containing aldehyde and ketone water extracts of samples (from left; five ampules each) TL10a, TL1, and TL4. All three Tagish Lake samples contained a dark nonpolar oily residue that was insoluble in water and that naturally separated from the solution as a film on the ampules' inner glass surfaces. The TL1 extract was particularly oily compared to those of TL10a and TL4.

TL1, but rather a process such as Michael addition or decarboxylation/deamination of dicarboxylic or diamino acids, which would result in relatively higher abundances of $\beta$ - and $\gamma$-amino acids, respectively (Elsila et al. 2016). The previously analyzed TL11h and TL5b samples are similarly enriched in $\beta$ - and $\gamma$-amino acids, 


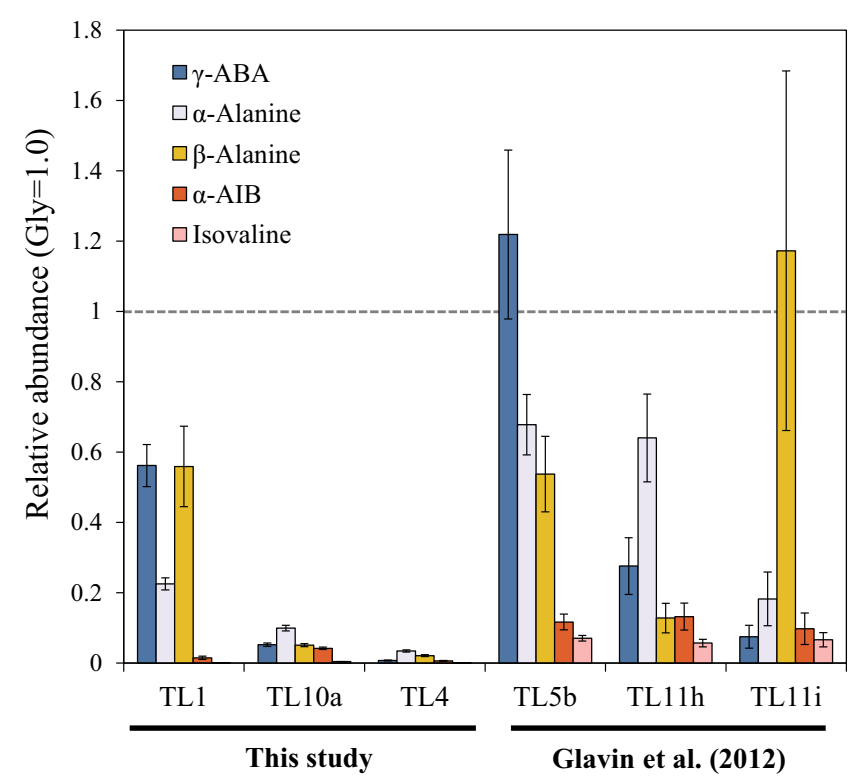

Fig. 5. Molar relative abundances $(\mathrm{Gly}=1.0)$ of amino acids in hydrolyzed samples of TL1, TL10a, and TL4 (this study) compared to abundances for hydrolyzed samples of TL5b, TL11h, and TL11i by Glavin et al. (2012). All values are blank-subtracted, with the exception of those of TL10a (see the Aldehydes and Ketones: Identification, Quantification, and $\delta 13 \mathrm{C}$ Analysis section for details).

and also contain relatively more $\alpha$ isomers, suggesting that they have recorded some additional input from Strecker synthesis type reactions.

Despite their similarly low total abundances of amino acids, TL4 and TL10a are unlike TL11i in terms of the relative abundances of glycine. Both TL4 and TL10a are dominated by glycine (at 90 and 70 mole $\%$, respectively), whereas glycine in TL11i only comprised $27 \mathrm{~mole} \%$ of the amino acid content, implying that the low abundances of amino acids in TL4 and TL10a resulted from distinct processes than those that affected the composition of TL11i. This observed difference in composition is perhaps unsurprising for TL4, as the TL4 specimen shows several characteristics (e.g., low abundance of phyllosilicates and high porosity) that suggest that it has experienced a unique alteration history (Gilmour et al. 2019). In contrast, the amino acid composition of TL10a is more difficult to explain given its overall similarity in mineral abundance to (and inferred degree of aqueous alteration with) specimen TL5b (Gilmour et al. 2019).

The amino acid compositions observed in the present study and previously for Tagish Lake samples exhibit some inconsistencies compared to general trends observed among carbonaceous chondrites. For example, the high relative abundances of $\gamma$-ABA and $\beta$-alanine observed in what are interpreted as moderately altered samples (TL1 and TL5b) is unusual; generally, higher relative abundances of $\beta$ - and $\gamma$-amino acids are considered indicators of higher levels of aqueous alteration (Botta et al. 2002; Glavin et al. 2011; Elsila et al. 2016; Modica et al. 2018). TL4 and TL10a are also unusual as their amino acid compositions are nearly identical, despite some clear differences in their organic contents and bulk properties. Our amino acid data suggest that aqueous alteration may not have been the sole factor influencing the type, extent, and duration of amino acid synthesis taking place in the Tagish Lake parent body asteroid and that perhaps other factors played a more significant role (e.g., the presence or absence of organic precursors). The mobilization and, thus, the availability of key precursor compounds (e.g., aldehydes/ketones, $\mathrm{HCN} / \mathrm{NH}_{4}$, aliphatic hydrocarbons) would have been directly affected by aqueous alteration processes, so these potential influences would have been interconnected to some extent. However, our results suggest that a particular degree of aqueous alteration does not necessarily dictate the types of amino acids that are synthesized in the Tagish Lake meteorite and that some mechanisms of amino acid synthesis may be decoupled from aqueous alteration processes.

The $\mathrm{D} / \mathrm{L}$ enantiomeric ratios for the amino acids in TL1, TL4, and TL10a (Table 3) are generally consistent with what has been observed previously for Tagish Lake samples; near-racemic values for alanine, $\beta$-ABA, and isovaline, and low $\mathrm{D} / \mathrm{L}$ ratios for glutamic acid, serine, and valine have been observed consistently across Tagish Lake specimens (Glavin et al. 2012). Without isotopic data for glutamic acid, serine, and valine in the present study, we are unable to fully evaluate whether the low $\mathrm{D} / \mathrm{L}$ ratios are inherent to the meteorite or whether they indicate a terrestrial input prior to sample analysis. However, the careful collection and curation history of the Tagish Lake meteorite support a meteoritic origin. The measured excesses of the L-enantiomer are potentially derived from extraterrestrial processes (e.g., amplification of conglomerate crystals; Glavin et al. 2012) and ${ }^{13} \mathrm{C}$ enriched values for amino acids with L-enantiomeric excesses's in previously analyzed Tagish Lake samples support this theory (Glavin et al. 2012). The relatively high $\mathrm{D} / \mathrm{L}$ ratios observed for aspartic acid in TL1 and TL4 in this study compared to previously analyzed specimens may reflect a lower degree of aqueous alteration experienced by these two samples, as L-enantiomeric excesses are thought to be amplified via aqueous alteration (Glavin and Dworkin 2009).

Figure 6 compares the compound-specific carbon isotope compositions of amino acids in TL1 with previous measurements from samples TL5b and TL11h (Glavin et al. 2012). Similar to the total amino acid 


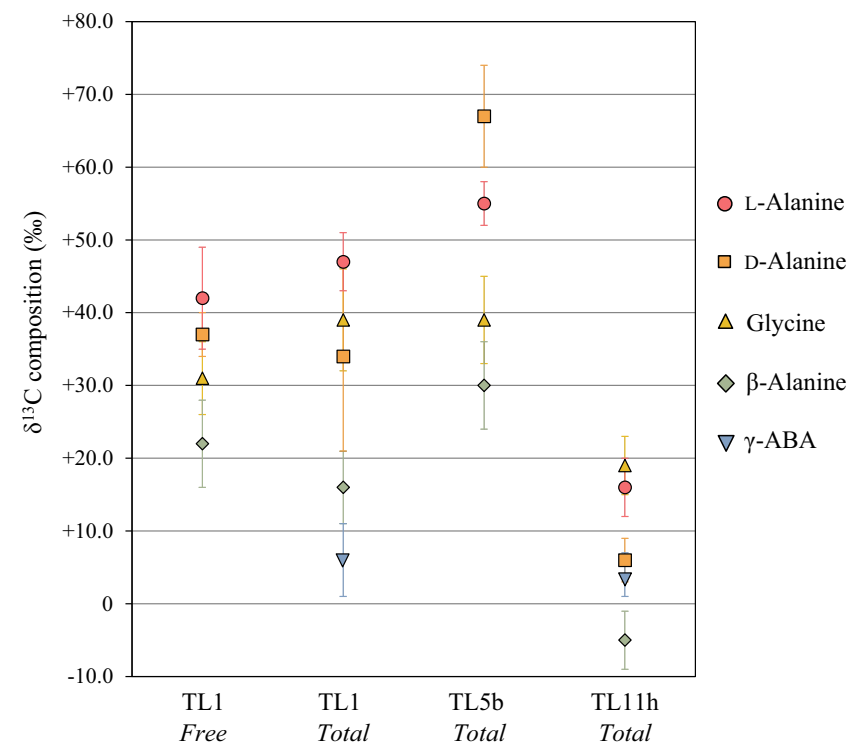

Fig. 6. Stable carbon isotope ratios for amino acids in hydrolyzed and nonhydrolyzed samples of TL1 (this study) compared to values reported by Glavin et al. (2012) for hydrolyzed samples of TL5b and TL11h.

abundances, several of the TL1 amino acid $\delta^{13} \mathrm{C}$ values fall intermediate between those of TL5b and TL11h. The $\delta^{13} \mathrm{C}$ values exhibit a general trend toward more ${ }^{13} \mathrm{C}$-depleted compositions from TL5b to TL1 to TL11h (Fig. 6). Exceptions to this trend are the glycine and hydrolyzed $\beta$-alanine values of TL5b which overlap with TL1 values within error, and the $\gamma$-ABA values for TL1 and TL11h, which also overlap within error. The differences in amino acid $\delta^{13} \mathrm{C}$ values between TL5b and TL11h have been previously discussed by Glavin et al. (2012); the more ${ }^{13} \mathrm{C}$-depleted values were interpreted as indicating more extensive aqueous alteration and/or a secondary pulse of alteration. However, there are other possible explanations for the differences observed. Relatively ${ }^{13} \mathrm{C}$-depleted amino acid compositions (e.g., in TL11h) could indicate a ${ }^{13} \mathrm{C}$ depletion of the source/precursor (e.g., ${ }^{13} \mathrm{C}$-depleted aldehydes, ketones, imines, and/or HCN). The relatively ${ }^{13} \mathrm{C}$-depleted amino acids in TL11h could also indicate a greater fractionation effect associated with the reaction due to lower temperature conditions (Sephton and Gilmour 2001). Alternatively, the TL11h amino acids may have been synthesized first, followed by TL1 and then TL5b; in theory, as preferentially ${ }^{13} \mathrm{C}$-depleted amino acids are synthesized (e.g., amino acids in TL11h), the isotopic composition of the Strecker source/precursor would shift toward a more ${ }^{13} \mathrm{C}$ enriched composition and the subsequently synthesized amino acids would reflect that shift (e.g., amino acids in TL1 and TL5b). This scenario, however, is only applicable if TL1, TL5b, and TL11h were all part of highly interconnected closed system. Last, the $\delta^{13} \mathrm{C}$ values could indicate that the amino acids in TL1 and $\mathrm{TL} 5 \mathrm{~b}$ were fractionated (shifted toward more ${ }^{13} \mathrm{C}$ enriched compositions) as ${ }^{12} \mathrm{C}$-containing amino acids were preferentially consumed or degraded during subsequent chemical reactions. The fact that the TL1 and TL11h $\gamma$-ABA values share a common carbon isotope composition is consistent with this theory, as the bulk of the $\gamma$-ABA detected in both specimens was released during hydrolysis, suggesting that the $\gamma$-ABA may have been minerally bound and not chemically available for postsynthetic reactions.

\section{The Origin of Aldehydes and Ketones in the Tagish Lake Meteorite}

The relative abundances of aldehydes and ketones in the Tagish Lake specimens (Table 4) are similar to the distribution observed previously for the Murchison meteorite (Simkus et al. 2019) although the $\delta^{13} \mathrm{C}$ values, ranging from $-51.5 \%$ to $-0.34 \%$, are highly ${ }^{13} \mathrm{C}$-depleted compared to those measured for Murchison $(-10.0 \%$ to $+66.4 \%$ ). The Tagish Lake formaldehyde values, ranging from $-51.5 \%$ to $-34.6 \%$, are the most ${ }^{13} \mathrm{C}$-depleted, even more so than the terrestrial formaldehyde $\delta^{13} \mathrm{C}$ value measured for the procedural blanks $(-28.1 \%$ oo $)$ The highly ${ }^{13} \mathrm{C}$-depleted values in Tagish Lake suggest that these compounds are not primordial, as interstellar aldehydes and ketones are expected to be highly ${ }^{13} \mathrm{C}$-enriched $\left(\delta^{13} \mathrm{C}\right.$ $>+117 \%$ ) based on ${ }^{12} \mathrm{C} /{ }^{13} \mathrm{C}$ measurements for interstellar formaldehyde $\quad\left(\delta^{13} \mathrm{C}=+117-4933 \%, \quad{ }^{12} \mathrm{C} /{ }^{13} \mathrm{C}=15-80\right.$; Wirström et al. 2012) and nearby molecular clouds $\left(\delta^{13} \mathrm{C}=+117-392 \%,{ }^{12} \mathrm{C} /{ }^{13} \mathrm{C}=+64-80 ; \quad\right.$ Henkel et al. $1980,1982)$. As was proposed for aldehydes and ketones in the Murchison meteorite, the meteoritic carbonyl compounds detected here are likely derived from secondary formation processes during aqueous alteration in the asteroid. The relative ${ }^{13} \mathrm{C}$-depletion of the Tagish Lake samples compared to the Murchison sample indicates one or more of the following. (1) The Tagish Lake alteration temperatures were lower, resulting in greater isotopic fractionations, (2) that Tagish Lake has experienced more excessive chemical processing via secondary aqueous alteration reactions, or (3) that the carbon measured in Murchison and Tagish Lake are derived from different starting pools.

If Strecker synthesis reactions involve significant isotope fractionations, the $\alpha$-amino acid products are expected to be relatively ${ }^{13} \mathrm{C}$-depleted in comparison to their aldehyde and ketone precursors, as the lighter isotope of carbon $\left({ }^{12} \mathrm{C}\right)$ would be preferentially converted to amino acids through the reaction. Based on their ${ }^{13} \mathrm{C}$-enriched values (Fig. 7), glycine and 


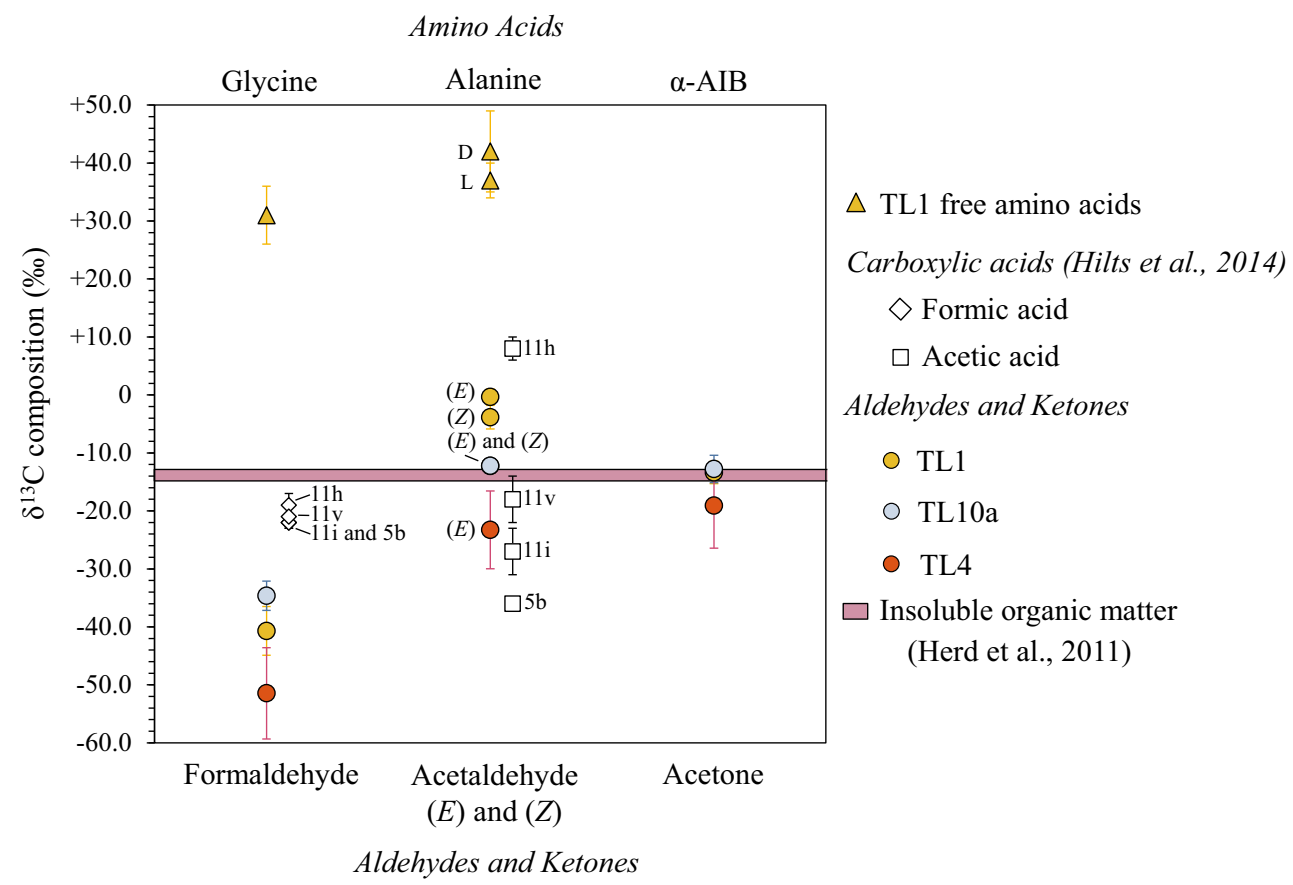

Fig. 7. Carbon stable isotope compositions (\% VPDB) of aldehydes and ketones in TL1, TL4, and TL10a (this study); the corresponding amino acids for TL1 (this study); the corresponding carboxylic acids from previously analyzed specimens (Hilts et al. 2014); and insoluble organic matter (Herd et al. 2011). Two acetaldehyde data points for a single Tagish Lake specimen represent the $(E)$ and $(Z)$ isomers. Two alanine data points represent the D- and L-enantiomers.

alanine detected in TL1 are not products of Strecker synthesis reactions involving the formaldehyde and acetaldehyde detected in the TL1 specimen. The aldehydes and ketones do not appear to be primordial, but rather are likely derived from secondary alteration reactions in the parent body asteroid. The isotopic separations between the TL1 amino acid $\delta^{13} \mathrm{C}$ ratios and the corresponding TL1 aldehyde and ketone $\delta^{13} \mathrm{C}$ ratios are large ( $\Delta$ [glycine-formaldehyde] $\sim 70 \%$ and $\Delta$ [alanine-acetaldehyde] $\sim 40 \%$ ), potentially representing isotopic fractionations associated with decomposition of amino acids in low-temperature conditions. Oxidation of the IOM associated with secondary aqueous alteration may be an additional source of meteoritic aldehydes and ketones (Cody and Alexander 2005). The differing amounts of the oily residue present in the Tagish Lake water extracts (Fig. 4) seem to correlate with the total abundances of aldehydes and ketones measured for each specimen, suggesting that there may be a relationship between the IOM/aromatic carbon content and carbonyl content for these samples.

\section{Variations in Monocarboxylic Acid Contents}

The monocarboxylic acid content of the Tagish Lake samples, specifically the abundance of acetic acid, may be used to group the samples into two categories, specimens with relatively high abundances of acetic acid (225-293 ppm; TL5b, TL1, and TL11h) and specimens with lower abundances of acetic acid (71-94 ppm; TL4, TL11i, and TL11v) (Herd et al. 2011; Hilts et al. 2014) (Table 5). The moderately altered specimens (TL1, TL5b, TL11h) exhibited the highest total abundances, while the minimally altered (TL4) and heavily altered (TL11i, TL11v) specimens yielded lower total abundances. These observations suggest that at least a proportion of the monocarboxylic acids are derived from alteration-driven reactions, such as oxidation of aldehydes (Corey et al. 1968) or oxidation of the macromolecular organic matter (Oba and Naraoka 2006). Extensive aqueous alteration (e.g., TL11i, TL11v), however, may have been detrimental to the preservation of these compounds.

Acetic acid is commonly the most abundant monocarboxylic acid detected in hot-water extracts of carbonaceous chondrite samples (Krishnamurthy et al. 1992; Naraoka et al. 1999; Huang et al. 2005; Oba and Naraoka 2006). The Tagish Lake specimens, however, exhibited relatively higher abundances of formic acid. This difference in relative abundances observed across studies may be a result of differences in methodologies and/or meteorite storage conditions (Hilts et al. 2014). 
TL1 and TL4 contain the highest abundances of formic acid observed to date for the Tagish Lake meteorite. The relatively high concentrations of formic acid detected in TL1 and TL4 may reflect the importance of maintaining low-temperature storage conditions during subsampling and powdering; the samples were kept cold right up until the water extraction step, including the powdering step. TL10a did not contain any detectable formic acid in either of the two water extracts suggesting that the specimen does not contain intrinsic formic acid. In fact, the bulk of the monocarboxylic acids detected in the TL10a samples may be terrestrial in origin based on comparisons with the procedural blanks, suggesting that TL10a does not contain intrinsic carboxylic acids, with the exception of benzoic acid and potentially a trace amount of acetic acid. These observations alone suggest that TL10a is a relatively highly altered sample and that the lower molecular weight carboxylic acids degraded and/or were mobilized during parent body aqueous alteration. The presence of benzoic acid is consistent with this theory, as it is relatively less labile/less soluble in water in comparison to the carboxylic acids detected and is less likely to be mobilized by water during periods of alteration. We also consider the possibility that the lack of monocarboxylic acids in TL10a is due to the absence of key precursor compounds (see the next section).

\section{Weakly Polar Aliphatic and Aromatic Compounds}

The DCM solvent extractions and analyses of TL1, TL4, and TL10a yielded two notable differences in their organic contents (Fig. 3, Table 6). (1) TL1 contained several PAHs (naphthalene, fluoranthene, pyrene), while free PAHs were absent from both TL4 and TL10a. This observation is consistent with the relatively higher abundance of a nonpolar, "oily" supernatant in water extracts of TL1 (Fig. 4) and (2) two separate DCM extractions (Fig. 3) revealed that the TL10a specimen has a unique composition that is essentially devoid of weakly polar aliphatic hydrocarbons.

The presence of PAHs in TL1 and the absence of PAHs in TL4 and TL10a may provide some important insight into the alteration history of these three samples. PAHs are most often discussed as having a preaccretionary origin, synthesized in low-temperature interstellar clouds or circumstellar environments prior to the formation of the solar system (Gilmour 2003). However, PAHs may also be derived from oxidation of the IOM during aqueous alteration on the parent body asteroid (Wing and Bada 1992). The absence of PAHs in TL4, which appears to be minimally aqueously altered, suggests that the PAHs in the Tagish Lake meteorite were primarily synthesized during aqueous alteration; if the PAHs were pre-accretionary in origin, we would expect them to be detectable in the minimally altered samples as well. For a comparison to other Tagish Lake lithologies, the previously analyzed TL5b specimen contained many PAHs (e.g., branched isomers of naphthalene, and pyrene), while TL11h contained naphthalene only, and TL11i contained no PAHs at all (Hilts et al. 2014). The presence of PAHs in the midsequence samples (TL1, TL5b, TL11h) and the absence of PAHs in the endmember samples (TL4, TL11i, and possibly TL10a) suggest that, similar to other organic compound classes, there may be a "sweet spot" for the production and preservation of free PAHs via aqueous alteration that is reflected in TL1, TL5b, and TL11h. The relatively high free PAH content observed for TL1, TL5b, and TL11h may have played a role in yielding alteration-derived organics (e.g., carboxylic acids or aldehydes/ketones) in these three specimens.

Collectively, the absence of weakly polar aliphatic hydrocarbons, PAHs, and intrinsic carboxylic acids, and the presence of amino acids, aldehydes, and ketones (in low abundances) in TL10a suggest that TL10a has a unique and unusual alteration history. The alteration processes in TL10a appear to have favored the production and/or preservation of amino acids and aldehydes/ketones over other compound classes. Without carbon isotope analyses for the amino acids in TL10a, it is difficult to assess the origins of these compounds and whether the amino acids, aldehydes, and ketones in TL10a share a common synthetic relationship. However, given the low soluble organic content of this specimen, we hypothesize that, at some point, TL10a experienced a pulse of aqueous alteration at elevated temperatures or with a high water/rock ratio that resulted in the degradation and/or mobilization of most soluble organics. If this is true, then the amino acids, aldehydes, and ketones detected in TL10a may have been introduced to TL10a via mobilization from another region of the asteroid. Alternatively, the amino acids may not be as directly influenced by aqueous alteration as previously thought; it is possible that these compounds were synthesized via a mechanism that does not require the presence of liquid water, though the presence of aldehydes and ketones in TL10a is more difficult to interpret without an alteration-driven reaction. Whatever the mechanisms involved, the disparity in the complement of soluble organic compounds between TL10a and mid-sequence specimens (e.g., TL5b, TL11h), coupled with the similarities in mineralogical indicators of degree of aqueous alteration (Gilmour et al. 2019), support the unique alteration history for this specimen.

The high abundances of soluble organics present in TL1 and the low abundances observed in TL4 support 
the idea of an optimal degree of aqueous alteration for producing and preserving high abundances of prebiotic compounds. Our data are also consistent with the parent body asteroid being an interconnected system that has experienced some fluid flow rather than static aqueous alteration, as we seem to have observed some loss or mobilization of organics across Tagish Lake specimens (e.g., TL10a). Further interpretation may require some evaluation of which specific factors determine the "degree" of parent body aqueous alteration, as variations in water/rock ratios, duration of alteration, and alteration temperatures may have very different effects on the soluble organic chemistry.

\section{CONCLUSIONS}

The three newly analyzed Tagish Lake specimens yielded unique soluble organic contents that suggest distinct parent body alteration histories. The distribution and abundances of amino acids, carboxylic acids, and weakly polar aliphatic and aromatic hydrocarbons in TL1 resembled the soluble organic contents of the previously analyzed TL5b and TL11h (Herd et al. 2011; Glavin et al. 2012; Hilts et al. 2014); TL1 exhibited relatively high abundances soluble organic compounds and appears to reflect a "sweet spot" of aqueous alteration that did not result in significant degradation or loss of alteration-derived organics. TL4, which appears to be a minimally altered sample, yielded very low abundances of organics, supporting the theory that many of the soluble organics observed in the Tagish Lake meteorite are primarily generated via alteration-driven reactions. In contrast, specimen TL10a lacks both monocarboxylic acids and weakly polar aliphatic and aromatic compounds, and contains low abundances of amino acids, aldehydes, and ketones, and has therefore experienced a higher degree of alteration and/or a distinct, soluble organicdepleted alteration history.

\section{ACKNOWLEDGMENTS}

D.N.S. and R.W.H. thank Drs Matthew Ross and Aaron Skelhorne, MacEwan University, for their assistance with GC-MS analyses. Funding for this study was provided by a Natural Sciences and Engineering Research Council (NSERC) Grant 261740 to C.D.K.H., an NSERC Canada Graduate Scholarship (CGS D3) to D.N.S., and an Izaak Walton Killam Memorial Scholarship to D.N.S. This research was also supported in part by a NASA Postdoctoral Program Fellowship to D.N.S. (NASA Goddard Space Flight Center, administered by Universities Space Research Association under contract with NASA), NASA's
Emerging Worlds Program (award 14-EW14_2-0010 to J.C.A.), the Planetary Science Division Internal Scientist Funding Program through the Fundamental Laboratory Research (FLaRe) work package to J.E.E., and the NASA Astrobiology Institute through funding awarded to the Goddard Center for Astrobiology under proposal 13-13NAI7-0032. The authors thank Dr. Yoko Kebukawa and one anonymous reviewer for their careful review of this manuscript.

\section{Editorial Handling-Dr. Scott Sandford}

\section{REFERENCES}

Alexander C. M. O’D., Cody G. D., Kebukawa Y., Bowden R., Fogel M. L., Kilcoyne A. L. D., Nittler L. R., and Herd C. D. K. 2014. Elemental, isotopic, and structural changes in Tagish Lake insoluble organic matter produced by parent body processes. Meteoritics \& Planetary Science 49:503-525.

Bland P. A., Cressey G., and Menzies O. N. 2004. Modal mineralogy of carbonaceous chondrites by X-ray diffraction and Mossbauer spectroscopy. Meteoritics \& Planetary Science 39:3-16.

Blinova A. I., Zega T. J., Herd C. D. K., and Stroud R. M. 2014a. Testing variations within the Tagish Lake meteorite -I: Mineralogy and petrology of pristine samples. Meteoritics \& Planetary Science 49:473-502.

Blinova A. I., Herd C. D. K., and Duke M. J. M. 2014 b. Testing variations within the Tagish Lake meteorite-II: Whole-rock geochemistry of pristine samples. Meteoritics \& Planetary Science 49:1100-1118.

Botta O., and Bada J. L. 2002. Extraterrestrial organic compounds in meteorites. Surveys in Geophysics 23:411-467.

Botta O., Glavin D. P., Kminek G., and Bada J. L. 2002. Relative amino acid concentrations as a signature for parent body processes of carbonaceous chondrites. Origins of Life and Evolution of the Biosphere 32:143-163.

Brown P. G., Hildebrand A. R., Zolensky M. E., Grady M., Clayton R. N., Mayeda T. K., Tagliaferri E., Spalding R., MacRae N. D., Hoffman E. L., Mittlefehldt D. W., Wacker J. F., Bird J. A., Campbell M. D., Carpenter R., Gingerich H., Glatiotis M., Greiner E., Mazur M. J., McCausland P. J. A., Plotkin H., and Mazur T. R. 2000. The fall, recovery, orbit, and composition of the Tagish Lake meteorite: A new type of carbonaceous chondrite. Science 290:320-325.

Cody G. D. and Alexander C. M. O'D. 2005. NMR studies of chemical structural variation of insoluble organic matter from different carbonaceous chondrite groups. Geochimica et Cosmochimica Acta 69: 1085-1097.

Corey E. J., Gilman N. W., and Ganem B. E. 1968. New methods for the oxidation of aldehydes to carboxylic acids and esters. Journal of the American Chemical Society 90:5616-5617.

Cronin J. R. 1976. Acid-labile amino acid precursors in the Murchison meteorite. Origins of Life 7:337-342.

Cronin J. R. and Chang S. 1993. Organic matter in meteorites: Molecular and isotopic analysis of the Murchison meteorite. In The chemistry of life's origins, edited by Greenberg J. M., Mendoza C. X., and Pirronelle V. Dordrecht, the Netherlands: Kluwer Academic Publishers. pp. 209-258. 
Docherty G., Jones V., and Evershed R. P. 2001. Practical and theoretical considerations in the gas chromatography/ combustion/isotope ratio mass spectrometry $\delta^{13} \mathrm{C}$ analysis of small polyfunctional compounds. Rapid Communications in Mass Spectrometry 15:730-738.

Ehrenfreund P., Glavin D. P., Botta O., Cooper G., and Bada J. L. 2001. Extraterrestrial amino acids in Orgueil and Ivuna: Tracing the parent body of CI type carbonaceous chondrites. Proceedings of the National Academy of Sciences 98:2138-2141.

Elsila J. E., Glavin D. P., and Dworkin J. P. 2009. Cometary glycine detected in samples returned by Starbust. Meteoritics \& Planetary Science 44:1323-1330.

Elsila J. E., Charnley S. B., Burton A. S., Glavin D. P., and Dworkin J. P. 2012. Compound-specific carbon, nitrogen, and hydrogen isotopic ratios for amino acids in CM and $\mathrm{CR}$ chondrites and their use in evaluating potential formation pathways. Meteoritics \& Planetary Sciences 47:1517-1536.

Elsila J. E., Aponte J. C., Blackmond D. G., Burton A. S., Dworkin J. P., and Glavin D. P. 2016. Meteoritic amino acids: Diversity in compositions reflects parent body histories. ACS Central Science 2:370-379.

Gilmour I. 2003. Structural and isotopic analysis of organic matter in carbonaceous chondrites. In Treatise on geochemistry, edited by Holland H. D. and Turekian K. K., vo1. 1. Amsterdam, The Netherlands: Elsevier. pp. 269-290.

Gilmour C. M., Herd C. D. K., and Beck P. 2019. Water abundance in the Tagish Lake meteorite from TGA and IR spectroscopy: Evaluation of aqueous alteration. Meteoritics \& Planetary Science. In Press.

Glavin D. P. and Dworkin J. P. 2009. Enrichment of the amino acid L-isovaline by aqueous alteration on CI and CM meteorite parent bodies. Proceedings of the National Academy of Sciences 106:5487-5492.

Glavin D. P., Dworkin J. P., Aubrey A., Botta O., Dotty J. H. III, Martins Z., and Bada J. L. 2006. Amino acid analyses of antarctic CM2 meteorites using liquid chromatography_time of flight-mass spectrometry. Meteoritics \& Planetary Science 41:899-902.

Glavin D. P., Callahan M. P., Dworkin J. P., and Elsila J. E. 2011. The effects of parent body processes on amino acids in carbonaceous chondrites. Meteoritics \& Planetary Science 45:1948-1972.

Glavin D. P., Elsila J. E., Burton A. S., Callahan M. P., Dworkin J. P., Hilts R. W., and Herd C. D. K. 2012. Unusual nonterrestrial L-proteinogenic amino acid excesses in the Tagish Lake meteorite. Meteoritics \& Planetary Science 47:1347-1364.

Glavin D. P., Alexander C. M. O’D., Aponte J. C., Dworkin J. P., Elsila J. E., and Yabuta H. 2018. The origin and evolution of organic matter in Carbonaceous chondrites and links to their parent bodies. In Primitive meteorites and asteroids, edited by Abreu N. Amsterdam, The Netherlands: Elsevier. pp. 205-271

Grady M. M., Verchovsky A. B., Franchi I. A., Wright I. P., and Pillinger C. T. 2002. Light element geochemistry of the Tagish Lake CI2 chondrite: Comparison with CI1 and CM2 meteorites. Meteoritics \& Planetary Science 37:713735.

Henkel C., Walmsley C. M., and Wilson T. L. 1980. Formaldehyde in giant molecular clouds: $\mathrm{H}_{2}$ densities and corrections to the $\left({ }^{12} \mathrm{C} /{ }^{13} \mathrm{C}\right)$ ratios. Astronomy and Astrophysics 82:41-47.
Henkel C., Wilson T. L., and Bieging J. 1982. Further $\left({ }^{12} \mathrm{C} /{ }^{13} \mathrm{C}\right)$ ratios from formaldehyde: A variation with distance from the galactic center. Astronomy \& Astrophysics 109:344-351.

Herd C. D. K., Blinova A., Simkus D. N., Huang Y., Tarozo R., Alexander C. M. O’D., Gyngard F., Nittler L. R., Cody G. D., Fogel M. L., Kebukawa Y., Kilcoyne A. L. D., Hilts R. W., Slater G. F., Glavin D. P., Dworkin J. P., Callahan M. P., Elsila J. E., De Gregorio B. T., and Stroud R. M. 2011. Origin and evolution of prebiotic organic matter as inferred from the Tagish Lake meteorite. Science 332, 1304-1307.

Herd C. D. K., Hilts R. W., Skelhorne A. W., and Simkus D. N. 2016. Cold curation of pristine astromaterials: Insights from the Tagish Lake meteorite. The Meteoritical Society 51:499-519.

Hilts R. W., Herd C. D. K., Simkus D. N., and Slater G. F. 2014. Soluble organic compounds in the Tagish Lake meteorite. Meteoritics \& Planetary Science 49:526-549.

Huang Y., Wang Y., Alexandre M. R., Lee T., Rose-Petruck C., Fuller M., and Pizzarello S. 2005. Molecular and compound-specific isotopic characterization of monocarboxylic acids in carbonaceous chondrites. Geochimica et Cosmochimica Acta 69:1073-1084.

Huber C. and Wächtershäuser G. 2003. Primordial reductive amination revisited. Tetrahedron Letters 44:1695-1697.

Hudson R. L., Lewis A. S., Moore M. H., Dworkin J. P., and Martin M. P. 2009. Enigmatic isovaline: Investigating the stability, racemization and formation of a non-biological meteoritic amino acid. ASP Conference Series 420:157-162.

Krishnamurthy R. V., Epstein S., Cronin J. R., Pizzarello S., and Yuen G. U. 1992. Isotopic and molecular analyses of hydrocarbons and monocarboxylic acids of the Murchison meteorite. Geochimica et Cosmochimica Acta 56:4045-4058.

Modica P., Martins Z., Meinert C., Zanda B., and d'Hendecourt L. L. S. 2018. The amino acid distribution in laboratory analogs of extraterrestrial organic matter: A comparison to CM chondrites. The Astrophysical Journal 865:1-11.

Naraoka H., Shimoyama A., and Harada K. 1999. Molecular distribution of monocarboxylic acids in Asuka carbonaceous chondrites from Antarctica. Origins of Life and Evolution of the Biosphere 29:187-201.

Oba Y. and Naraoka H. 2006. Carbon isotopic composition of acetic acid generated by hydrous pyrolysis of macromolecular organic matter from the Murchison meteorite. Meteoritics \& Planetary Science 41:1175-1181.

O’Brien D. M., Fogel M. L., and Boggs C. L. 2002. Renewable and nonrenewable resources: Amino acid turnover and allocation to reproduction in Lepidoptera. Proceedings of the National Academy of Sciences 99:4413-4418.

Peltzer E. T., Bada J. L., Schlesinger G., and Miller S. L. 1984. The chemical conditions on the parent body of the Murchison meteorite: Some conclusions based on amino, hydroxy, and dicarboxylic acids. Advanced Space Research 4:69-74.

Pietrucci F., Aponte J. C., Starr R., Pérez-Villa A., Elsila J. E., Dworkin J. P., and Saitta A. M. 2018. Hydrothermal decomposition of amino acids and origins of prebiotic meteoritic organic compounds. ACS Earth \& Space Chemistry 2:588-598.

Pizzarello S., Huang Y., Becker L., Poreda R. J., Nieman R. A., Cooper G., and Williams M. 2001. The organic content of the Tagish Lake meteorite. Science 293:2236-2239. 
Ralchenko M., Britt D. T., Samson C., Herd C. D. K., Herd R. K., and McCausland P. J. A. 2014. Bulk physical properties of the Tagish Lake meteorite frozen pristine fragments (abstract \#1021). 14th Lunar and Planetary Science Conference. CD-ROM.

Sephton M. A. 2002. Organic compounds in carbonaceous meteorites. Natural Product Reports 19:292-311.

Sephton M. A. and Gilmour I. 2001. Compound-specific isotope analysis of the organic constituents in carbonaceous chondrites. Mass Spectrometry Reviews 20:111-120.

Simkus D. N., Aponte J. C., Hilts R. W., Elsila J. E., and Herd C. D. K. 2019. Compound-specific carbon isotope compositions of aldehydes and ketones in the Murchison

\section{SUPPORTING INFORMATION}

Additional supporting information may be found in the online version of this article:

Data S1. Data points for Fig. 1.

Data S2. Data points for Data. 2. meteorite. Meteoritics \& Planetary Science 54:142:156. https://doi.org/10.1111/maps.13202.

Wing M. R. and Bada J. L. 1992. The origin of the polycyclic aromatic hydrocarbons in meteorites. Origins of Life and Evolution of the Biosphere 21:375-383.

Wirström E. S., Charnley S. B., Geppert W. D., and Persson C. M. 2012. Observations of carbon isotopic fractionation in interstellar formaldehyde (abstract \#1611). 43rd Lunar and Planetary Science Conference. CD-ROM.

Zolensky M. E., Nakamura K., Gounelle M., Mikouchi T., Kasama T., Tachikawa O., and Tonui E. 2002. Mineralogy of Tagish Lake: An ungrouped type 2 carbonaceous chondrite. Meteoritics \& Planetary Science 37:737-761.

Data S3. Data points for Fig. 3.

Data S4. Data points for Fig. 5.

Data S5. Data points for Fig. 6.

Data S6. Data points for Fig. 7. 